\title{
Epithelial-specific A2B adenosine receptor signaling protects the colonic epithelial barrier during acute colitis
}

\author{
CM Aherne ${ }^{1,2}$, B Saeedi ${ }^{2}$, CB Collins ${ }^{2,3}$, JC Masterson ${ }^{2,4}$, EN McNamee ${ }^{1,2}$, L Perrenoud $^{1,2}$, CR Rapp $^{1,2}$, \\ VF Curtis $^{2}$, A Bayless ${ }^{2}$, A Fletcher ${ }^{5}$, LE Glover ${ }^{2}$, CM Evans $^{5}$, P Jedlicka ${ }^{6}$, GT Furuta ${ }^{2,4}$, EF de Zoeten ${ }^{2,3}$, \\ SP Colgan ${ }^{2}$ and HK Eltzschig ${ }^{1}$
}

Central to inflammatory bowel disease (IBD) pathogenesis is loss of mucosal barrier function. Emerging evidence implicates extracellular adenosine signaling in attenuating mucosal inflammation. We hypothesized that adenosinemediated protection from intestinal barrier dysfunction involves tissue-specific signaling through the A2B adenosine receptor (Adora2b) at the intestinal mucosal surface. To address this hypothesis, we combined pharmacologic studies and studies in mice with global or tissue-specific deletion of the Adora2b receptor. Adora2 $b^{-1-}$ mice experienced a significantly heightened severity of colitis, associated with a more acute onset of disease and loss of intestinal epithelial barrier function. Comparison of mice with Adora2b deletion on vascular endothelial cells (Adora2 $b^{f / f}{ }^{\text {VeCadCre }}{ }^{+}$) or intestinal epithelia (Adora2 $b^{f / f /}$ VillinCre ${ }^{+}$) revealed a selective role for epithelial Adora2b signaling in attenuating colonic inflammation. In vitro studies with Adora2b knockdown in intestinal epithelial cultures or pharmacologic studies highlighted Adora2b-driven phosphorylation of vasodilator-stimulated phosphoprotein (VASP) as a specific barrier repair response. Similarly, in vivo studies in genetic mouse models or treatment studies with an Adora2b agonist (BAY 60-6583) recapitulate these findings. Taken together, our results suggest that intestinal epithelial Adora2b signaling provides protection during intestinal inflammation via enhancing mucosal barrier responses.

\section{INTRODUCTION}

Inflammatory bowel disease (IBD) including Crohn's disease (CD) and ulcerative colitis (UC) are relapsing-remitting conditions characterized by uncontrolled intestinal inflammation and tissue destruction. ${ }^{1}$ A recent study points to the increasing incidence and prevalence of both $\mathrm{CD}$ and $\mathrm{UC}$ in North America, highlighting the urgent need for effective therapeutic options. ${ }^{2}$ Genome-wide association studies have established a firm link between dysfunction in host-microbial responses and aberrant mucosal barrier protection with the development of IBD. ${ }^{1}$ As such, greater understanding of dysregulated mucosal homeostasis in IBD may provide novel therapeutic options.

Release of extracellular adenosine was previously implicated as an endogenous protective response during mucosal inflammation (reviewed in Aherne et al. ${ }^{3}$ ). Adenosine acts through a number of cell surface receptors to mediate its signaling responses. ${ }^{3}$ Studies to date have outlined a role for the A2A (A2A adenosine receptor (Adora2a) $)^{4-7}$ and A2B (Adora2b) ${ }^{8-13}$ adenosine receptors in acute inflammation. However, the functional consequences of adenosine release and signaling during mucosal inflammation observed in IBD remains to be

\footnotetext{
${ }^{1}$ Department of Anesthesiology, School of Medicine, University of Colorado Anschutz Medical Campus, Aurora, Colorado, USA. ${ }^{2}$ Mucosal Inflammation Program, School of Medicine, University of Colorado Anschutz Medical Campus, Aurora, Colorado, USA. ${ }^{3}$ Section of Pediatric Gastroenterology, Hepatology and Nutrition, Digestive Health Institute, Children's Hospital Colorado, Aurora, Colorado, USA. ${ }^{4}$ Gastrointestinal Eosinophilic Diseases Program, Section of Pediatric Gastroenterology, Hepatology and Nutrition, Digestive Health Institute, Children's Hospital Colorado, Aurora, Colorado, USA. ${ }^{5}$ Division of Pulmonary Sciences and Critical Care Medicine, School of Medicine, University of Colorado Anschutz Medical Campus, Aurora, Colorado, USA and ${ }^{6}$ Department of Pathology, School of Medicine, University of Colorado Anschutz Medical Campus, Aurora, Colorado, USA. Correspondence: CM Aherne (carol.aherne@ucdenver.edu)
} 
defined. Previous observations indicate that generation of extracellular adenosine in models of IBD is profoundly protective. ${ }^{14-16}$ Adenosine signaling through Adora2a protects the intestinal mucosa in a chronic model of IBD; ${ }^{17}$ however, the role of the Adora2b receptor remains undefined. Recent findings highlight a tissue-protective role for Adora $2 \mathrm{~b}$ signaling during acute intestinal inflammation as occurs in IBD. ${ }^{12,18}$ These studies suggest that Adora2b expression at the intestinal epithelium is a key protective signaling pathway during acute inflammation. However, in separate studies, intestinal epithelial Adora2b signaling was observed to be deleterious in models of IBD. ${ }^{19-21}$ Reasons for these divergent findings have yet to elucidated, but may be due in part to genetic background differences in mice studied or in experimental design. We postulated that Adora2b functions in a tissue-specific manner at the mucosal surface to mediate protection in intestinal inflammation. Our aim was to investigate the function of Adora2b signaling at the mucosal surface during acute intestinal inflammation as experienced during IBD. To examine this, we generated novel mouse lines with tissue-specific deletion of the Adora2b receptor. Our studies demonstrated that mice with global knockout of Adora2b $\left(\right.$ Adora $2 b^{-1-}$ ) experience rapid, early onset of acute DSS (dextran sulfate sodium) colitis associated with premature loss of mucosal barrier function. Tissue-specific murine studies established a definitive role for intestinal epithelial Adora2b expression in driving mucosal protection during acute colitis. Supported by in vitro findings with Adora2b intestinal epithelial knockdown (KD) cells, we have outlined the Adora2b signaling pathway as a directly protective mechanism in repairing the damaged mucosal barrier through phosphorylation of vasodilator-stimulated phosphoprotein (VASP) in the intestinal epithelium.

Taken together, we observed that tissue-specific expression of the Adora2b receptor on the intestinal epithelium is an endogenous protective response to maintain mucosal barrier function during acute colitis. Pharmacological studies demonstrate that targeting the Adora $2 \mathrm{~b}$ receptor mediates mucosal protection from acute intestinal inflammation. This points to the exciting possibility of exploiting Adora2b signaling in a tissue-specific manner as a therapeutic approach in IBD.

\section{RESULTS}

\section{Mice deficient in Adora2b demonstrate early onset and} increased severity of DSS colitis

The exact role of the Adora2b receptor in IBD remains to be defined. In previous studies, Adora $2 \mathrm{~b}$ signaling on the intestinal epithelium was implicated as the relevant signaling pathway. ${ }^{12,19-21}$ However, these studies do not agree on whether Adora2b has a beneficial or deleterious role in models of IBD. We hypothesized that previous divergent findings may be due to distinct roles for Adora2b on different stromal tissue. To investigate this, we assessed mice with whole-body deletion of Adora2b (Adora2 $b^{-1-}$ ) over the course of DSS colitis. Decreased survival of Adora $2 b^{-1-}$ mice compared with wildtype controls $\left(\right.$ Adora $\left.2 b^{+/+}\right)$was noted when studies were performed using 4.5\% DSS (Supplementary Figure S1 online). Owing to high mortality rates in Adora2 $b^{-1-}$ mice at this percentage, we performed subsequent studies with 3-3.5\% DSS. Monitoring mice during the course of acute colitis revealed increased weight loss in Adora $2 b^{-1-}$ mice compared with wild-type controls (Figure 1a). Adora2 $b^{-1-}$ mice exhibited significantly greater colonic shortening (Figure $\mathbf{1 b}$ ) and loss of epithelial barrier function (Figure 1c), as measured by FITC (fluorescein isothiocyanate)-dextran permeability, compared with controls, from as early as day 3 following DSS. Upon termination of the study, Adora $2 b^{-1-}$ mice demonstrated enhanced neutrophil numbers in the colon (Figure 1d) and dramatically increased severity of histological disease (Figure 1e, f). These findings indicate that deficiency of Adora $2 b$ results in earlier onset of tissue damage and loss of mucosal barrier function during DSS.

\section{Vascular endothelial expression of Adora2b does not have a role in acute colitis}

Drawing from our findings in Adora $2 b^{-1-}$ mice (Figure 1), we investigated a tissue-specific role for stromal expression of the Adora $2 \mathrm{~b}$ receptor using newly developed tissue-specific knockout mice. A previous study implicated vascular endothelial Adora $2 \mathrm{~b}$ as a key regulator of acute inflammation. ${ }^{8}$ Mice with deletion of Adora $2 b$ in vascular endothelial cells (Adora $2 b^{f l f l} \mathrm{VeCadCre}^{+}$) and their wild-type controls $\left(\mathrm{VeCadCre}^{+}\right)$were exposed to DSS. Genotyping PCR of tails (not shown) and whole colonic tissues (Supplementary Figure S2b) demonstrated Cre and Flox expression in desired tissue. Deletion of Adora2b in the vascular endothelium had no effect on acute inflammation experienced during DSS, as evidenced by no differences in weight loss, colon length, and histological damage between Adora $2 b^{f l / f l}$ VeCadCre $^{+}$and $\mathrm{VeCadCre}^{+}$mice (Figure 2a-d). These findings indicate that Adora2b expression on the vascular endothelium has no significant role in acute intestinal inflammation as observed in IBD.

\section{Specific deletion of intestinal epithelial Adora2b results in increased severity of acute colitis}

Having observed no significant impact of vascular endothelialderived Adora2b on the outcome of DSS colitis, we investigated the function of intestinal epithelial-expressed Adora $2 \mathrm{~b}$ during acute colitis. Previous findings indicate that Adora2b is expressed to a high level on intestinal epithelial cells and its expression is induced during colonic inflammation. ${ }^{22}$ We generated mice with deletion of Adora $2 b$ in intestinal epithelial cells (Adora2b $b^{f l f l}$ VillinCre $^{+}$) and exposed them along with wild-type controls (VillinCre ${ }^{+}$) to DSS. Genotyping PCR of tails (not shown) and colonic intestinal epithelial cells (described in Flow Cytometry methods: Supplementary Figure S2c) demonstrated Cre and Flox expression in desired tissue. Adora $2 b^{f l f f l}$ VillinCre $^{+}$mice exhibited significantly increased weight loss during the course of DSS compared with VillinCre ${ }^{+}$ controls (Figure 3a). Mirroring our findings in whole-body knockout studies (Figure 1), Adora2 $b^{f l / f l} V_{\text {VillinCre }}{ }^{+}$mice experienced significantly greater colonic shortening and enhanced epithelial barrier permeability at early time points 

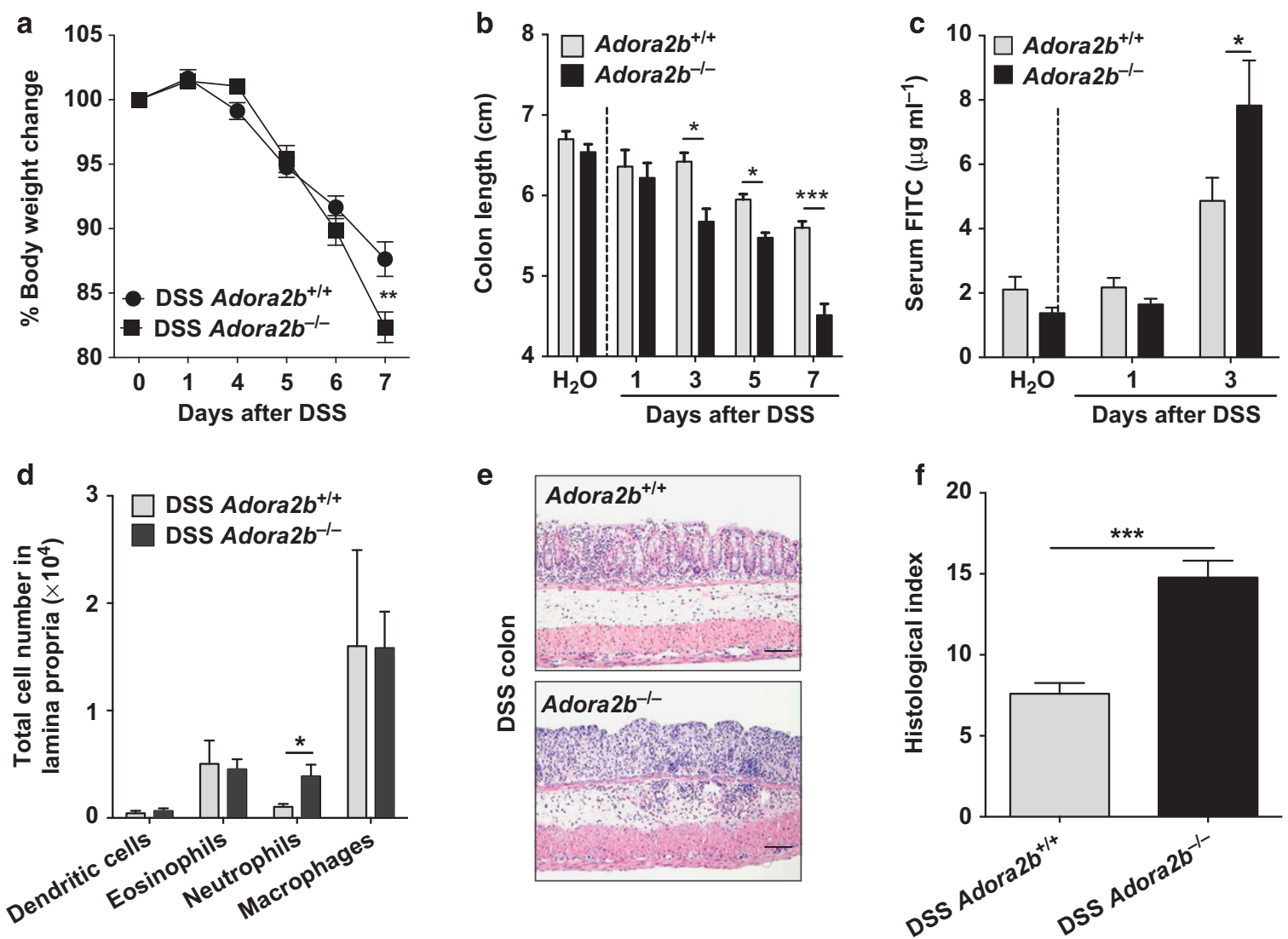

Figure 1 Adora2b (A2B adenosine receptor)-deficient mice experience early onset of acute colitis. Gender-, age-, and weight-matched Adora2bdeficient mice (Adora2 $b^{-1-}$ ) or C57BL/6 wild-type controls (Adora2 $b^{+/+}$) were exposed to dextran sulfate sodium (DSS) (3-3.5\%) over a time course of 7 days. (a) Daily weight measurements were obtained for each group of mice. (b) Following killing, colons were harvested and measured. Results are representative of one to two independent experiments with $n=4-13$ mice per group. (c) Mice were administered FITC (fluorescein isothiocyanate)dextran by oral gavage $\left(0.6 \mathrm{mg} \mathrm{g}^{-1}\right.$ at $\left.80 \mathrm{mg} \mathrm{ml}^{-1}\right) 4 \mathrm{~h}$ before killing. Serum was harvested at killing (days 1 and 3 after DSS) and fluoresence measurement was used to determine FITC levels; $n=2-9$ mice per group from one independent experiment per time point. (d) Following colon harvest at day 7 after DSS, colonic lamina propria leukocytes were isolated and flow cytometry determined the frequency of GR-1 ${ }^{+}$(neutrophils), SiglecF ${ }^{+}$ (eosinophils), $\mathrm{F} 4 / 80^{+}$(macrophages), and $\mathrm{MHClI}{ }^{+} \mathrm{CD} 11 \mathrm{c}^{\mathrm{Hi}}$ (dendritic cells) cells. Actual cell number for each cell type was calculated based on the frequency of cell type multiplied by cell counts following organ harvest. Results are representative of one independent experiment with $n=3-4$ mice per group. (e) Representative histological sections from whole colon of Adora2 $b^{-1-}$ or Adora2 $b^{+1+}$ mice harvested following 7 days DSS. Bar $=100 \mu$; images acquired at original magnification $\times 10$. (f) Blinded histological analysis of whole colon from each group following DSS. Data represent 8-10 mice per group. Unless stated otherwise, results are representative of two independent experiments with 3-10 mice per group and are displayed as mean \pm s.e.m. Two-way analysis of variance (ANOVA) with post hoc Bonferroni $t$-test was used to determine statistical weight change, but in all other cases Student's $t$-test was used. ${ }^{\star} P<0.5 ;{ }^{\star \star} P<0.001 ;{ }^{* \star \star} P<0.0001$.

following DSS compared with VillinCre ${ }^{+}$controls (Figure $3 \mathbf{b}$, c, respectively). Tissue cytokine analysis revealed a trend towards an increase in cytokine levels in the tissue of Adora $2 b^{f l f l}$ VillinCre ${ }^{+}$mice compared with controls, particularly in interleukin-6 (IL-6) (Figure 3d). Finally, histological analysis of the distal colon (Figure $3 \mathbf{e}, \mathbf{f}$ ) demonstrated that Adora $2 b^{f l f l} V_{\text {VillinCre }}^{+}$mice experienced a significantly worse outcome of DSS colitis compared with wild-type controls. Our observations highlight a tissue-specific role for expression of Adora2b on intestinal epithelial cells as a protective response during the acute phase of intestinal inflammation.

\section{An Adora2b-specific agonist is protective during acute colitis}

Our studies reveal that endogenous expression of Adora2b on the intestinal epithelium is a protective mechanism during acute mucosal inflammation. However, the effect of an exogenous Adora2b agonist during acute colitis has not been investigated. We undertook studies using a specific Adora $2 \mathrm{~b}$ agonist (BAY 60-6583). This agonist has previously been demonstrated to have a high degree of specificity for the Adora $2 \mathrm{~b}$ receptor. ${ }^{23,24} \mathrm{We}$ confirmed its ability to induce the second messenger cAMP, a known downstream signaling molecule of the Adora2b receptor in both $\mathrm{CHO}$ (Chinese hamster ovarian) cells expressing the human Adora2b receptor and T84 intestinal epithelial cells (Supplementary Figure S4). Continuous administration of the Adora2b agonist provided potent tissue protection as measured by weight loss, colonic shortening, tissue permeability, tissue cytokine levels, and histological damage of the distal colon in acute DSS colitis compared with vehicle-treated controls (Figure 4a-f, respectively). This provides the first evidence 

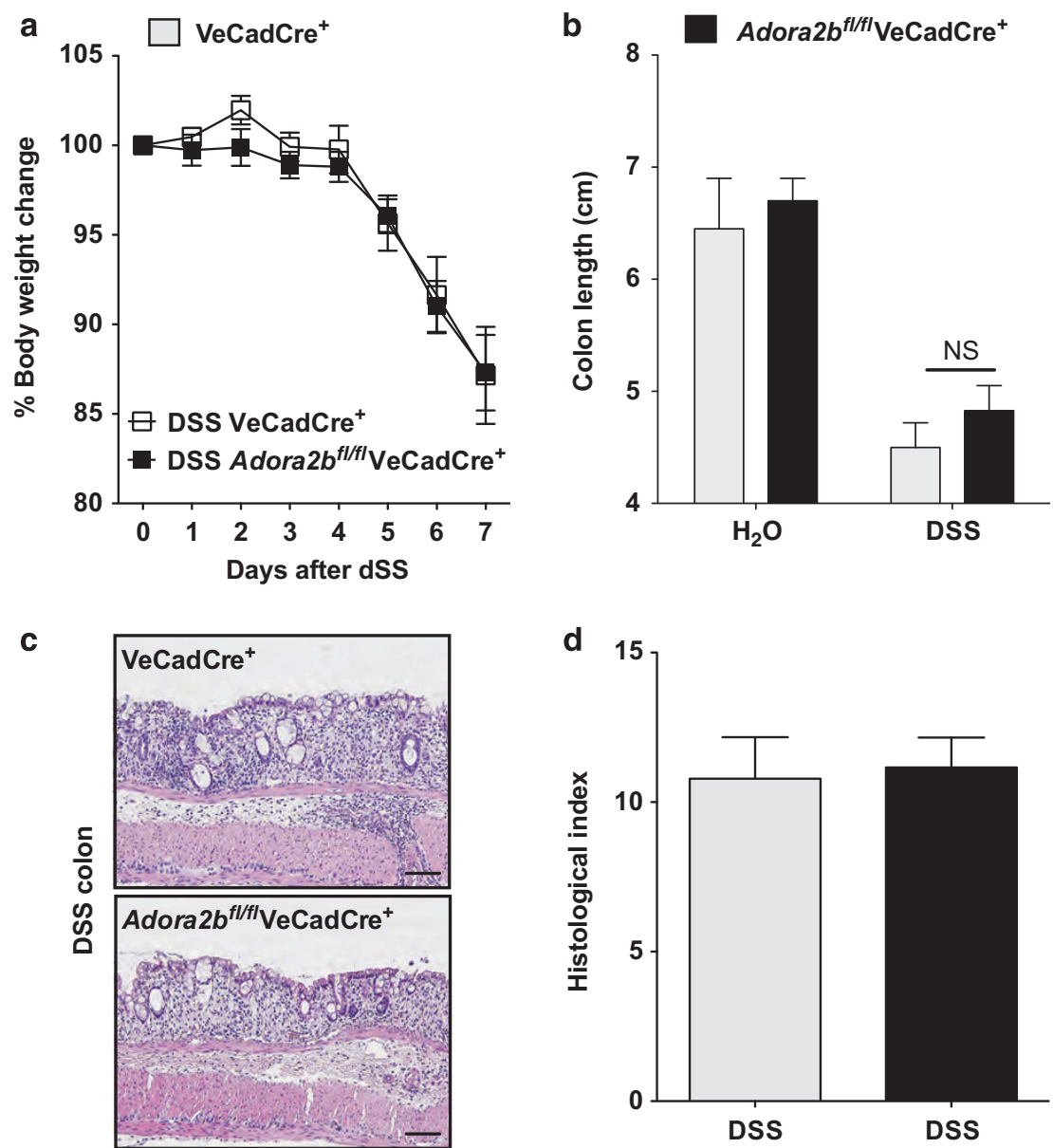

Figure 2 Adora2b (A2B adenosine receptor) in the vascular endothelium does not contribute to DSS (dextran sulfate sodium) colitis disease activity. Mice with vascular endothelial specific deletion of Adora2b (Adora2 $b^{f / f} / \mathrm{VeCadCre}{ }^{+}$) were generated and exposed to water or DSS $(3 \%)$ along with their wild-type Cre controls $\left(\mathrm{VeCadCre}^{+}\right.$). Following 7 days, mice were killed and the whole colon harvested by blunt dissection. (a) Daily weight measurements were taken for each group. (b) Upon harvest colon length was measured. (c) Representative whole colonic histological sections are displayed. Bar $=100 \mu \mathrm{m}$; images acquired at original magnification $\times 10$. (d) Blinded histological analysis of whole colon from each group following DSS. Results are representative of 7-8 mice per DSS group and 2 mice per water group, and are displayed as mean \pm s.e.m.

that an Adora2b agonist can provide mucosal protection in a model of IBD.

\section{Adora2b signaling does not effect epithelial barrier breakdown}

To date, we observed premature onset of acute colonic inflammation in mice deficient in the Adora $2 b$ receptor during DSS colitis (Figure 1). Tissue-specific deletion identified Adora2b expression on the intestinal epithelium as the key protective signaling pathway in this model (Figure 3). Considering that intestinal barrier permeability was significantly enhanced early in the course of DSS in both Adora2b ${ }^{-1-}$ (Figure 1c) and Adora $2 b^{f l f l} V_{\text {VillinCre }}^{+}$mice (Figure 3c), we sought to determine how intestinal epithelial Adora2b might modulate epithelial barrier breakdown.

A number of intrinsic and extrinsic mechanisms have been identified that regulate epithelial barrier breakdown during acute colitis. These include cytokine-induced epithelial barrier dysfunction, ${ }^{25}$ alterations in the phosphorylation status of myosin light chain (MLC), ${ }^{26}$ nuclear factor- $\kappa \mathrm{B}(\mathrm{NF}-\kappa \mathrm{B})$ activity, ${ }^{27}$ intestinal epithelial cell apoptosis, ${ }^{28}$ and mucin-2 expression. ${ }^{29}$ First, we analyzed the effect of Adora2b signaling on cytokine-induced epithelial barrier breakdown (Figure 5a). Barrier studies were performed in T84 intestinal epithelial cells as they express high levels of the Adora2b receptor ${ }^{22}$ in comparison with other epithelial cell lines that have been used to study Adora2b receptor function (Supplementary Figure S5a). ${ }^{30}$ T84 intestinal epithelial monolayers were cotreated with the Adora $2 \mathrm{~b}$ agonist or vehicle and a mixture of tumor necrosis factor- $\alpha(\mathrm{TNF} \alpha)$, IL- $1 \beta$, and interferon $\gamma$ (IFN $\gamma$ ) ("cytomix"). Following $72 \mathrm{~h}$ of treatment, a FITC-flux assay was performed to determine the flux rate across the barrier and thereby the permeability of the monolayer. Adora $2 b$ agonist treatment did not suppress the cytokine-induced increase in barrier permeability (Figure 5a).

The phosphorylation status of MLC has been demonstrated to be intimately involved in epithelial tight junction paracellular permeability ${ }^{31}$ with a recent study highlighting a key role for MLC phosphorylation in intestinal permeability in IBD. ${ }^{26}$ Treatment of polarized T84 intestinal epithelial monolayers for 


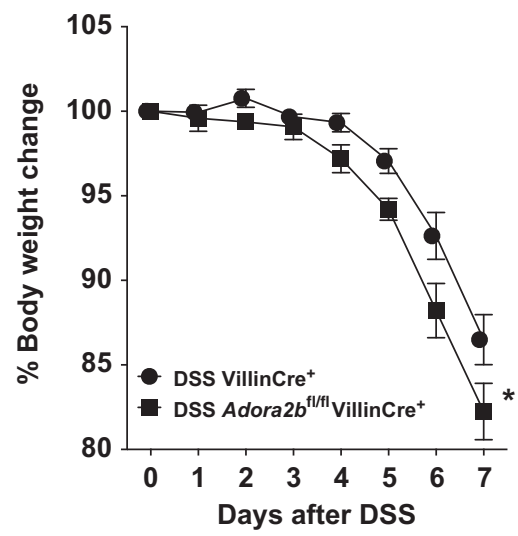

d

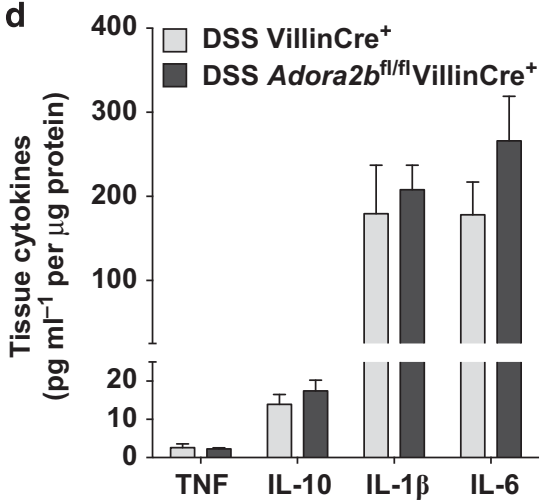

b

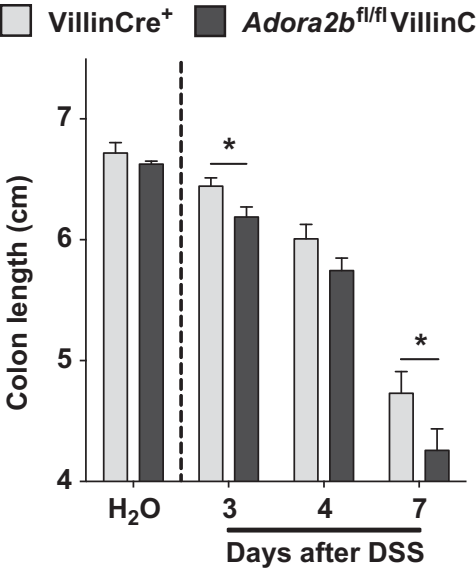

e

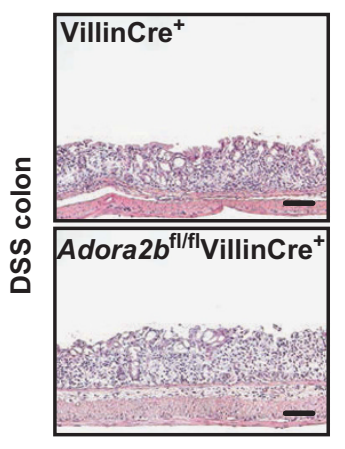

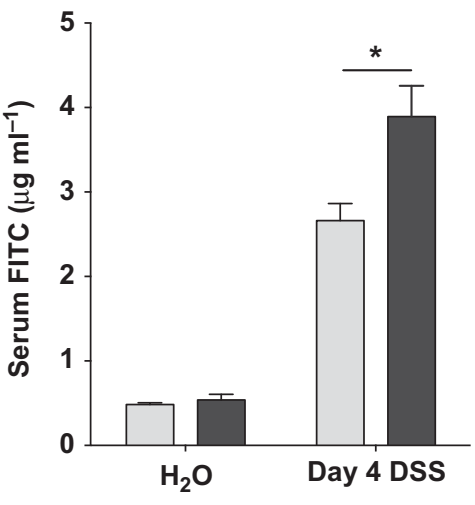

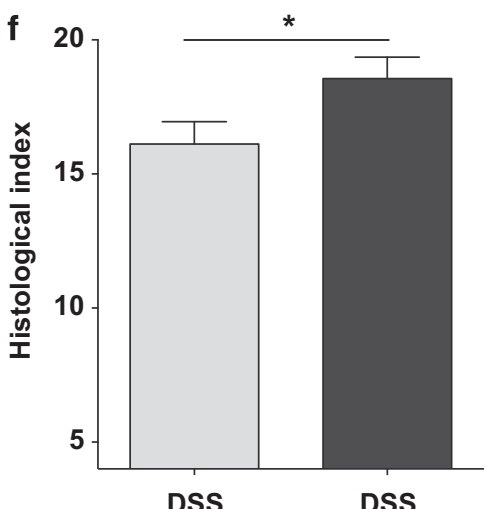

Figure 3 Epithelial-specific deletion of the Adora2b (A2B adenosine receptor) receptor results in significantly increased susceptibility to acute colitis. Mice with intestinal epithelial specific deletion of Adora2b (Adora2 $b^{f / / f}$ VillinCre $^{+}$) were generated and exposed to water or DSS (dextran sulfate sodium, 3-3.5\%) along with their wild-type Cre controls (VillinCre ${ }^{+}$). Mice were killed at 3, 4, and 7 days after DSS, and colons harvested by blunt dissection. (a) Daily weight measurements were assessed for each group of mice. (b) Following harvest at day 3, 4, or 7 colon lengths were measured. Results are representative of one to two independent experiments per time point with 4-18 mice per group. (c) On day 4 after DSS, FITC (fluorescein isothiocyanate)dextran was administered by oral gavage $\left(0.6 \mathrm{mg} \mathrm{g}^{-1}\right.$ at $\left.80 \mathrm{mg} \mathrm{m}^{-1}\right) 4 \mathrm{~h}$ before killing and serum collection. Fluorescence measurement was used to determine FITC levels; $n=3-8$ mice per group from one experiment. (d) Following harvest at day 7 after DSS, colon tissue was homogenized and tissue cytokines were measured by Meso Scale (Meso Scale Discovery, Rockville, MD). Results are displayed normalized to protein content and are representative of 4-6 mice per group. (e) Representative histological sections from distal colon of Adora2b $b^{f / f l}$ VillinCre $^{+}$or VillinCre $^{+}$mice harvested following 7 days DSS. Bar $=100 \mu \mathrm{m}$; images are acquired at original magnification $\times 10$. (f) Bar graph of blinded histological scoring of the distal colon following 7 days of DSS; $n=7-10$ mice per group. Unless stated otherwise, results are representative of two to three independent experiments with $4-10$ mice per DSS group and are displayed as mean \pm s.e.m. Two-way analysis of variance (ANOVA) with post hoc Bonferroni $t$-test was used to determine statistical weight change, but in all other cases Student's $t$-test was used. ${ }^{*} P<0.05$.

30 min (Figure $5 \mathbf{b}$ ) or 180 min (not shown) did not alter MLC phosphorylation. Of note, an experiment performed with polarized Caco-2 cells yielded similar findings (not shown).

The master transcription factor NF- $\kappa \mathrm{B}$ has a controversial role in regulating intestinal epithelial barrier function in acute colitis. ${ }^{27}$ Studies suggest that adenosine signaling through the Adora2b receptor inhibits NF- $\mathrm{KB}$ transcriptional activity. ${ }^{24,30,32}$ In line with previous findings, ${ }^{33}$ we observed no effect of cotreatment with the Adora2b agonist on TNF $\alpha$ - (Figure 5c) or IL-1 $\beta$ - (not shown) induced NF- $\kappa B$ transcriptional activity in intestinal epithelial cells using an NF- $\mathrm{KB}$-driven luciferase reporter.

Increased epithelial cell apoptosis may result in loss of epithelial barrier function during DSS colitis. $^{28,34}$ TUNEL (terminal deoxynucleotidyl transferase-mediated deoxyuridine triphosphate nick end labeling) staining revealed no differences in the number of apoptotic epithelial cells in the colon of Adora $2 b^{f l f l}$ VillinCre $^{+}$mice compared with VillinCre ${ }^{+}$controls at the time point when enhanced barrier dysfunction was observed during DSS (Figure 5d).

Finally, expression of the mucin, Muc-2, has been demonstrated to be essential in maintaining intestinal epithelial barrier function. ${ }^{29}$ Previous findings suggest that adenosine signaling can upregulate Muc-2 in epithelial cells. ${ }^{35}$ We observed no appreciable difference in Muc-2 expression in Adora2b ${ }^{-1-}$ mice or Adora2 $b^{f l f l} V_{\text {VillinCre }}{ }^{+}$mice compared with their controls at the time point when barrier dysfunction occurred (Supplementary Figure S3). Taken together, these findings suggest that the intestinal epithelial Adora2b receptor does not protect the epithelial barrier by suppressing cytokine-induced 
a
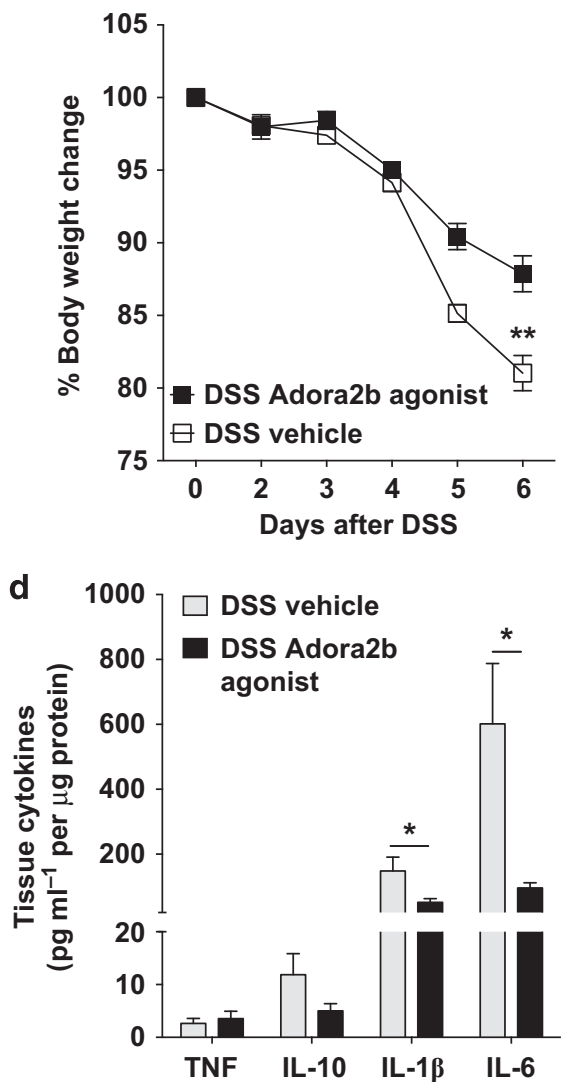

b

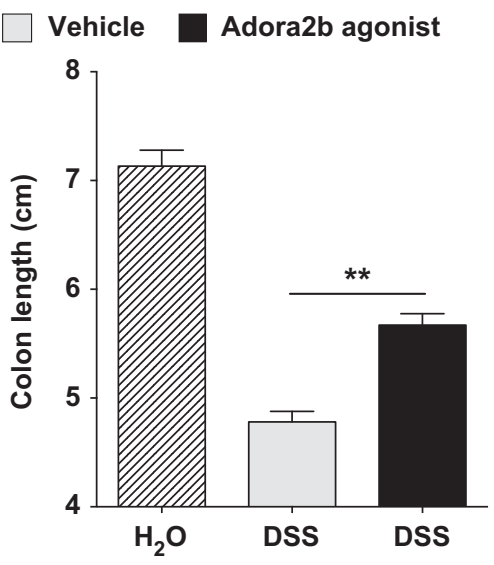

e

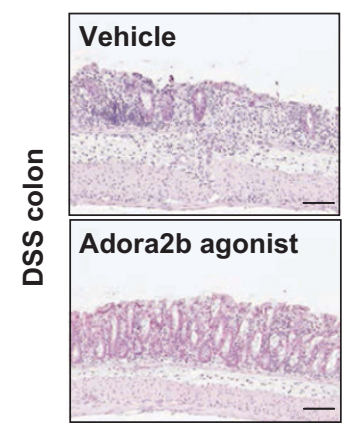

C
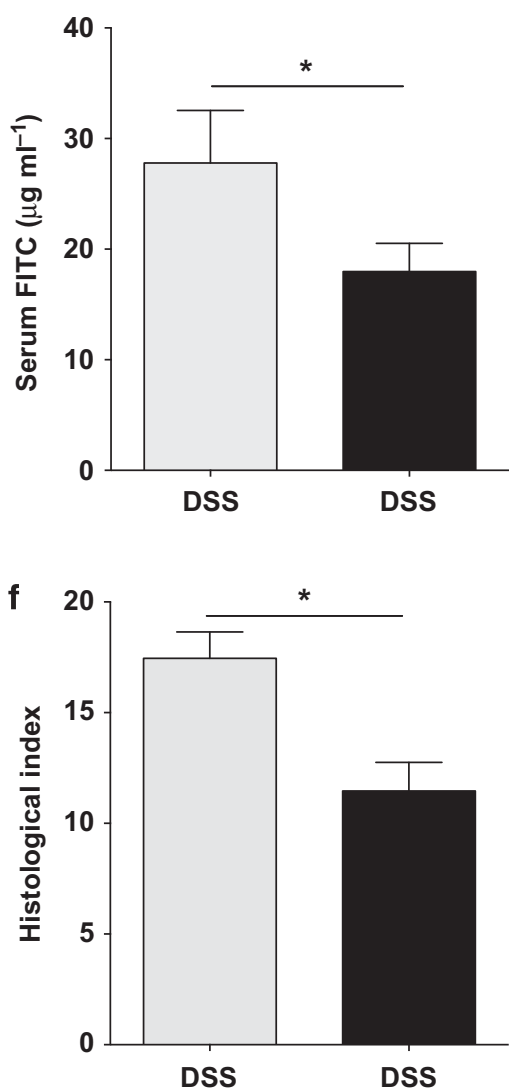

Figure 4 Treatment with an Adora2b (A2B adenosine receptor) agonist mediates mucosal protection observed in a model of acute colitis. Gender-, age-, and weight-matched C57BL/6 mice were treated with an Adora2b-specific agonist (BAY 60-6583; $1.2-1.25 \mathrm{mg} \mathrm{kg}^{-1}$ per day) or vehicle (30\% Solutol HS15 in $0.9 \%$ saline) using a subcutaneous osmotic pump beginning 1 day before administration of DSS (dextran sulfate sodium, 3.5-4\%) for 6 days. Mice exposed to DSS were orally gavaged with FITC (fluorescein isothiocyanate)-dextran $\left(0.6 \mathrm{mg} \mathrm{g}^{-1}\right.$ at $80 \mathrm{mg} \mathrm{ml}^{-1}$ ) $4 \mathrm{~h} \mathrm{before} \mathrm{killing} \mathrm{on} \mathrm{day} 6$, serum collection, and blunt dissection of the colon. (a) Daily weight measurements were obtained for each group of mice. (b) Colon lengths were measured upon harvest. (c) Fluorescence measurement determined FITC levels in the serum on day 6 after DSS. (d) Following harvest, cytokines in colonic tissue were measured by Meso Scale. Results are displayed normalized to protein content and represent 4-5 mice per group. (e) Representative colonic histological images from vehicle and Adora2b agonist-treated mice exposed to DSS. Bar $=100 \mu \mathrm{m}$; images were acquired at original magnification $\times 10$. (f) Bar graph of blinded histological scoring of the distal colon from vehicle and Adora2b agonist-treated mice exposed to DSS. Results are displayed as mean \pm s.e.m. and are representative of one to two independent experiments with 3-7 mice per group. Two-way analysis of variance (ANOVA) with post hoc Bonferroni $t$-test was used to determine statistical weight change, but in all other cases Student's $t$-test was used. ${ }^{\star} P<0.5 ;{ }^{\star \star} P<0.001$.

epithelial barrier permeability, by altering MLC phosphorylation status, by altering NF- $\mathrm{KB}$ transcriptional activity, or by affecting either intestinal epithelial cell apoptosis or Muc-2 expression.

\section{Adora2b regulates epithelial barrier resealing}

Having observed that Adora2b signaling was not effective in altering mechanisms associated with enhanced epithelial barrier breakdown, we directed our studies to look at alternate mechanisms by which Adora2b receptor signaling could improve epithelial barrier function. To mimic our in vivo model, we genetically deleted Adora2b in T84 intestinal epithelial cells in vitro (Supplementary Figure S5b). We used a calcium switch assay involving chelation of calcium with EDTA, which has been demonstrated to cause junction disassembly and a rapid increase in epithelial barrier permeability that is recoverable by the reintroduction of calcium. ${ }^{36,37}$ This allows for the assessment of epithelial junction regulation during acute disruption of the barrier such as that observed in DSS. Importantly, this assay allows us to assess the ability of the epithelial barrier to recover. Barrier recovery was monitored by measuring the rate at which a FITC-labeled dextran moved from the apical to the basolateral aspect of epithelial monolayers once calcium was reintroduced (Figure 6). The method by which this rate was calculated is discussed in Supplementary Materials. The rate at which control knockdown (KD) cells or vehicle-treated cells repair is defined as 1 and data are displayed as a fold change relative to this rate to account for interexperiment variability. Adora $2 b \mathrm{KD}$ cells demonstrated a significantly slower recovery of barrier function compared with their control KD cells (Figure 6a). A previous study implicated the phosphorylation of the focal adhesion protein VASP at $\mathrm{Ser}^{157}$ in cAMP-induced epithelial barrier repair following acute disruption. ${ }^{37}$ One of the major intracellular pathways downstream of the Adora2b receptor is the cAMP-protein 

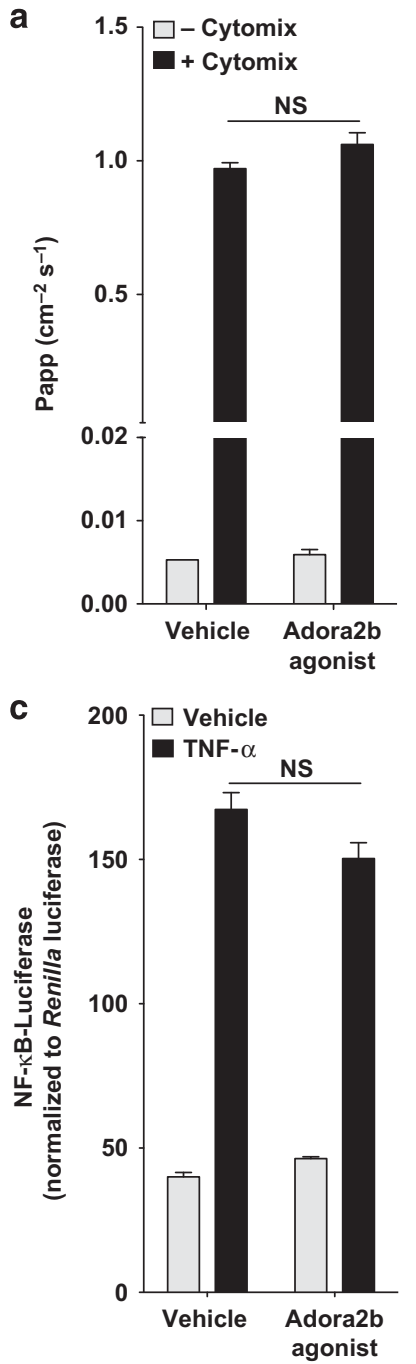

b
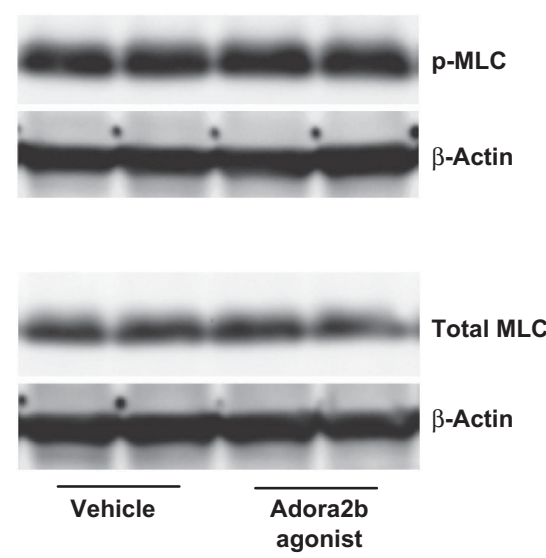

d

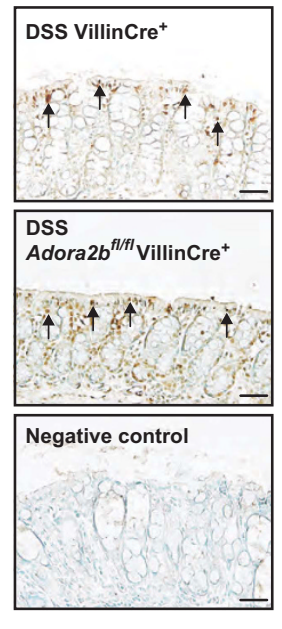

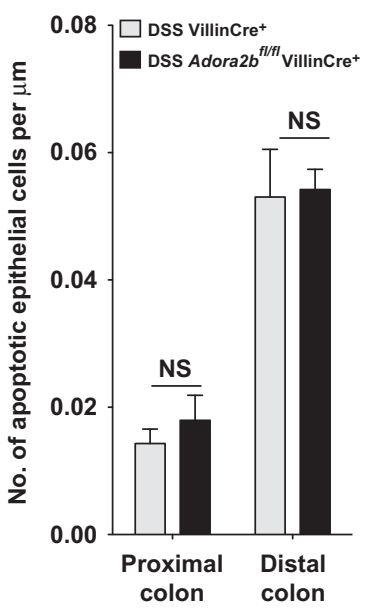

Figure 5 Epithelial Adora2b (A2B adenosine receptor) signaling does not affect epithelial barrier breakdown. (a) A combination of tumor necrosis factor$\alpha(\mathrm{TNF} \alpha)$, interleukin-1 $\beta$ (IL-1 $\beta$ ), and interferon $\gamma(\mathrm{IFN} \gamma)\left(\right.$ all $10 \mathrm{ng} \mathrm{ml}^{-1}$ ) or media alone was added to the basolateral aspect of polarized T84 intestinal epithelial cells. Vehicle or the Adora2b agonist (BAY 60-6583; $10 \mu \mathrm{m}$ ) was added to both chambers. Following $72 \mathrm{~h}$, FITC (fluorescein isothiocyanate)labeled dextran $(3 \mathrm{kDa})$ was added to the apical chamber and a flux assay was performed. The permeability of the monolayer was assessed by measuring the concentration of FITC in the basolateral chamber over time, calculated as the apparent permeability (Papp: $\mathrm{cm}^{-2} \mathrm{~s}^{-1}$ ). Results are representative of three independent experiments with 2-4 wells per group. (b) Confluent T84 intestinal epithelial cells were treated on the basolateral and apical aspect with vehicle or Adora2b-specific agonist (BAY $60-6583 ; 10 \mu \mathrm{m}$ ) for $30 \mathrm{~min}$ before cell harvest and total protein extraction. $\mathrm{p}-\mathrm{MLC}$ (myosin light chain) $\left(\right.$ Ser ${ }^{19}$ ), total MLC, and $\beta$-actin levels were determined by Western blot analysis. Results are representative of three independent experiments with $2-3$ wells per experiment. (c) Caco-2 intestinal epithelial cells were transfected with an equal amount of a nuclear factor- $\kappa \mathrm{B}(\mathrm{NF}-\mathrm{kB})$-responsive promoter attached to a firefly luciferase reporter (NF-kB-luciferase) and a control reporter vector (Renilla luciferase). Cells were treated in triplicate for $6 \mathrm{~h}$ with tumor necrosis factor- $\alpha$ (TNF- $\alpha)\left(10 \mathrm{ng} \mathrm{ml}^{-1}\right)$ and the Adora2b agonist (BAY 60-6583; $\left.10 \mu \mathrm{M}\right)$. Equal volumes of cell lysate were assayed for luciferase activity. Data were normalized to Renilla luciferase and are representative of three independent experiments. (d) Mice with intestinal epithelial specific deletion of $A d o r a 2 b$ $\left(\right.$ Adora2 $b^{f l / f}$ VillinCre ${ }^{+}$) were exposed to water or DSS (dextran sulfate sodium, $3 \%$ ) along with their wild-type Cre controls (VillinCre ${ }^{+}$) for 4 days and colons harvested by blunt dissection. Representative images are displayed of apoptotic colonic epithelial cells identified by TUNEL (terminal deoxynucleotidyl transferase-mediated deoxyuridine triphosphate nick end labeling) staining of paraffin-embedded colonic sections. Arrows point to positively stained cells. Bar $=50 \mu \mathrm{m}$; images were acquired at original magnification $\times 20$. Terminal deoxynucleotidyl transferase was omitted as a negative control. Bar graph of the number of apoptotic colonic epithelial cells in the proximal or distal colon of each group of mice. The number is displayed relative to the length of basement membrane. Scoring was carried out in a blinded manner in three randomly selected mice per group of 6 , with a total of six to eight sections per mouse. All results are displayed as the mean \pm s.e.m. Statistical analysis was performed using the Student's $t$-test.

kinase A (PKA) pathway. ${ }^{3}$ We have demonstrated that Adora2b signaling rapidly enhances cAMP levels in T84 intestinal epithelial cells (within $30 \mathrm{~min}$ : Supplementary Figure S4c). Within $60 \mathrm{~min}$ of calcium reintroduction the epithelial barrier rapidly recovers. ${ }^{37}$ In this instance, a marked increase in VASP phosphorylation at $\mathrm{Ser}^{157}$ was observed by
60 min, which diminished thereafter. ${ }^{37}$ We monitored protein levels of p-VASP $\left(\mathrm{Ser}^{157}\right.$ ) and total VASP in our cells at the indicated time points of recovery (Figure 6b). Of note, the total VASP antibody also recognizes the phosphorylated form of VASP, confirming our results with p-VASP $\left(\mathrm{Ser}^{157}\right)$ antibody. Western blot confirmed that VASP is phosphorylated at Ser ${ }^{157}$ 
a

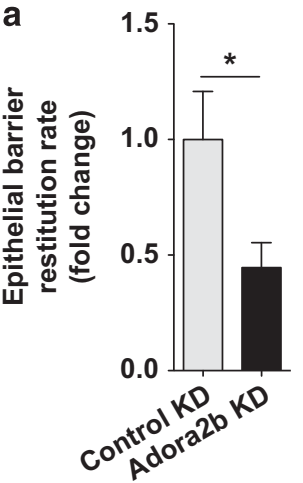

c

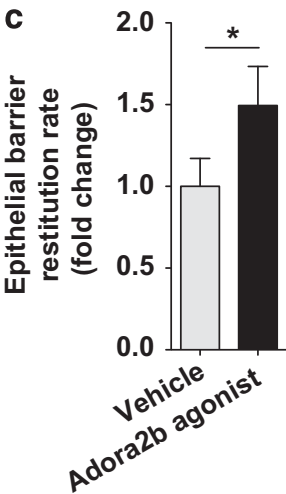

b

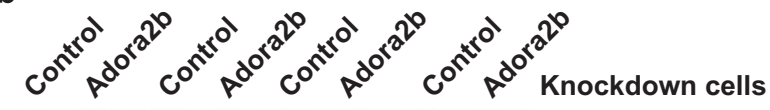

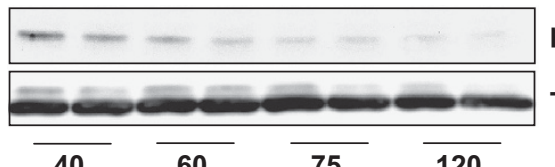

p-VASP

Total VASP

Time after $\mathrm{Ca}^{2+}$ switch (min)

d

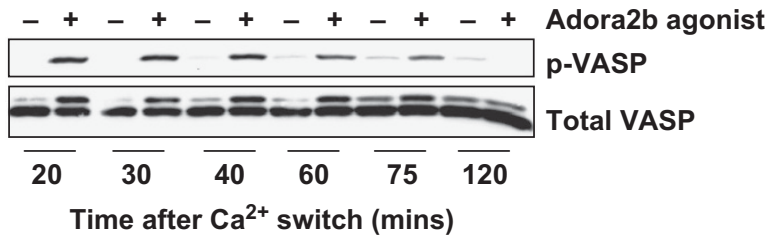

e

p-VASP
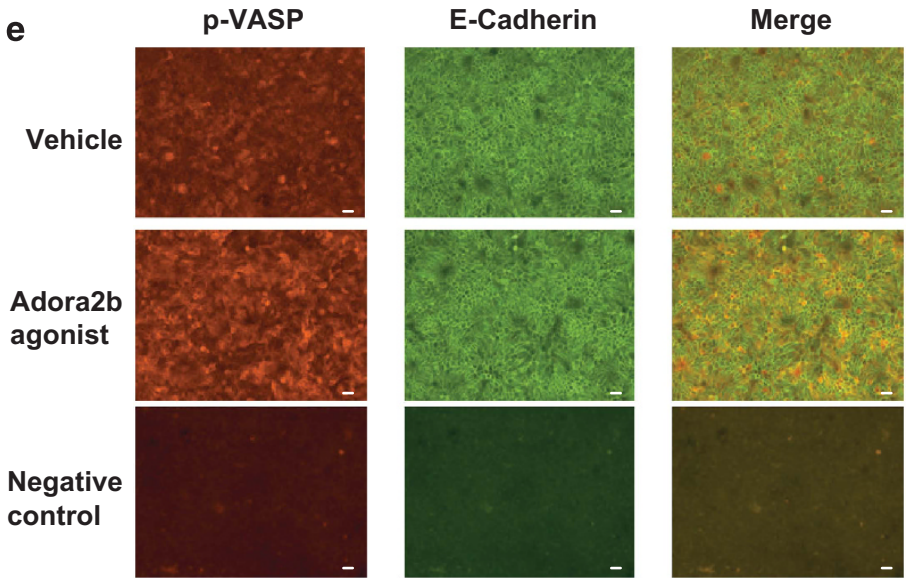

Figure 6 Epithelial Adora2b (A2B adenosine receptor) promotes barrier restitution in intestinal epithelial cells. Calcium switch assays were performed using intestinal epithelial cells with a fully competent barrier. Cells were placed in calcium-depleting conditions until barrier function was fully compromised and subsequently allowed to recover in calcium complete media for the time points outlined. (a) Barrier recovery studies were performed with T84 intestinal epithelial cells with constitutive Adora2b knockdown (Adora2b KD) or nonspecific gene KD (control KD) as mentioned. FITC (fluorescein isothiocyanate)-labeled dextran $(10 \mathrm{kDa})$ was added to the apical aspect of the cells at the start of the recovery period. Media from the basolateral aspect were sampled every $15 \mathrm{~min}$ for $105 \mathrm{~min}$ and fluorescence in the media at each point was measured to analyze rate of barrier restitution. Results are displayed as fold change in the rate of restitution and represent two independent experiments with 4-6 wells per experiment. (b) Constitutive Adora2b or control KD cells were used in calcium switch assays as described above and allowed to recover for the indicated times. Total protein was extracted and phosphorylated vasodilator-stimulated phosphoprotein ( $\mathrm{p}$-VASP) $\left(\mathrm{Ser}^{157}\right)$ and total VASP levels were determined by Western blot analysis. Results are representative of three independent experiments with 2 wells per time point per experiment. (c) T84 intestinal epithelial cells were used in calcium switch assays. Vehicle or the Adora2b-specific agonist (BAY 60-6583; $10 \mu \mathrm{m}$ ) was added to both the basolateral and apical aspects of the cells at the beginning of the recovery period. Barrier restitution rate was determined as described in a. Results are representative of three independent experiments with 4-6 wells per experiment. (d) T84 intestinal epithelial cells were treated as described in c and cells were harvested at different time points during the recovery period as indicated. Total protein was extracted and p-VASP $\left(\mathrm{Ser}^{157}\right)$ and total VASP levels were determined by Western blot analysis. Results are representative of three independent experiments with 2 wells per time point per experiment. (e) Calcium switch assays were performed with T84 cells as described in c. Cells were allowed to recover for $30 \mathrm{~min}$ in the presence of vehicle or Adora2b agonist before fixation with $2 \%$ paraformaldehyde. Cells were costained with a p-VASP $\left(\right.$ Ser $^{157}$ ) and an E-cadherin antibody followed by fluorescent secondary antibody labeling. Primary antibodies were omitted as negative control. Representative images were acquired at original magnification $\times 20$. Bar $=50 \mu \mathrm{m}$. Results are representative of three independent experiments with at least 2 wells per group. Results are displayed as mean \pm s.e.m. Statistical analysis was performed using the Student's $t$-test. ${ }^{\star} P<0.05$. 
during the first $60 \mathrm{~min}$ of barrier recovery, as observed previously (Figure 6b) ${ }^{37}$ Importantly, Adora2b KD cells were unable to phosphorylate VASP $\left(\operatorname{Ser}^{157}\right)$ to the same level as their control cells at time points analyzed and did not exhibit any alteration in the timecourse of VASP $\left(\mathrm{Ser}^{157}\right)$ phosphorylation. In contrast, T84 wild-type cells treated from the onset of recovery with an Adora2b-specific agonist (BAY 60-6583; $10 \mu \mathrm{M}$ ) exhibited an almost $40 \%$ increase in the rate of barrier recovery compared with vehicle-treated controls (Figure 6c). We noted a very rapid and sustained elevation of p-VASP $\left(\mathrm{Ser}^{157}\right)$ in agonist-treated cells over that in vehicle-treated controls during barrier recovery (Figure 6d). The position at which VASP is detected by Western blot makes loading quantification hard to determine. Therefore, our Western blot data demonstrate a qualitative trend in alteration of VASP phosphorylation as a consequence of Adora $2 \mathrm{~b}$ signaling. Agonist specificity for Adora2b-induced VASP (Ser ${ }^{157}$ ) phosphorylation was confirmed by pretreatment of T84 cells with a specific Adora $2 \mathrm{~b}$ antagonist before agonist cotreatment. Under these conditions, the Adora2b agonist failed to phosphorylate VASP (Ser ${ }^{157}$ ) (Supplementary Figure S6). Finally, we confirmed our Western blot findings by immunofluorescence staining (Figure 6e). We observed a substantial portion of p-VASP $\left(\mathrm{Ser}^{157}\right.$ ) localizing to E-cadherin-positive T84 epithelial cell junctions within 30 min of Adora2b agonist treatment during barrier recovery compared with vehicle controls (Figure 6e). This is consistent with a more rapid rate of barrier recovery in agonist-treated cells (Figure 6c). These results outline a direct effect of Adora2b signaling on epithelial barrier function, which our results suggest is mediated through the phosphorylation of VASP $\left(\operatorname{Ser}^{157}\right)$.

\section{PKA activity mediates Adora2b-driven VASP $\left(\mathrm{Ser}^{157}\right)$ phosphorylation}

PKA has been directly implicated in epithelial barrier recovery and VASP $\left(\right.$ Ser $\left.^{157}\right)$ phosphorylation. ${ }^{37}$ PKA is a known downstream mediator of Adora2b signaling. ${ }^{3}$ Treating T84 cells with the PKA inhibitor $(\mathrm{H} 89 ; 30 \mu \mathrm{M})$ before administration of the Adora2b-specific agonist (BAY 60-6583; 1 and $10 \mu \mathrm{M}$ ) revealed a concentration-dependent increase in p-VASP $\left(\mathrm{Ser}^{157}\right)$ that was significantly attenuated by the inhibition of PKA (Figure 7a). Analyzing the Adora2b signaling pathway during barrier recovery revealed a profound effect of PKA inhibition on the ability of the Adora2b agonist to phosphorylate VASP $\left(\operatorname{Ser}^{157}\right)$ (Figure 7b). We conclude that Adora2b signaling through PKA plays a significant role in VASP $\left(\mathrm{Ser}^{157}\right.$ ) phosphorylation during barrier recovery.

\section{Epithelial VASP $\left(\operatorname{Ser}^{157}\right.$ ) phosphorylation is significantly diminished in mice deficient in the epithelial Adora2b receptor during DSS}

To assess the relevance of p-VASP $\left(\mathrm{Ser}^{157}\right)$ in our in vivo model, colonic tissue from Adora2b $b^{f l f l} \mathrm{VillinCre}^{+}$and VillinCre ${ }^{+}$ mice was stained with p-VASP ( $\mathrm{Ser}^{157}$; red) and an epithelialspecific antibody (E-cadherin; green) at 4 and 7 days after DSS (Figure 8a, b). 4',6-diamidino-2-phenylindole (DAPI) (blue) served as a nuclear counterstain. Of note, the predominant a
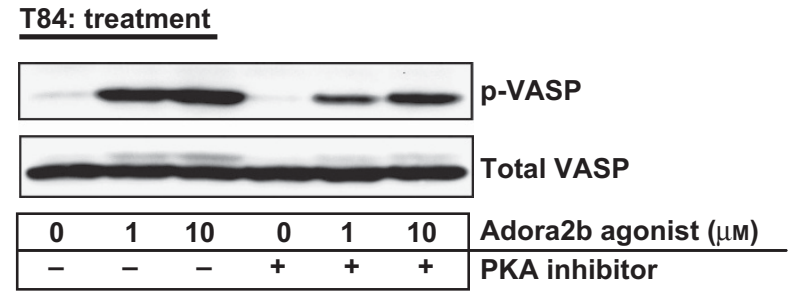

b T84: calcium switch

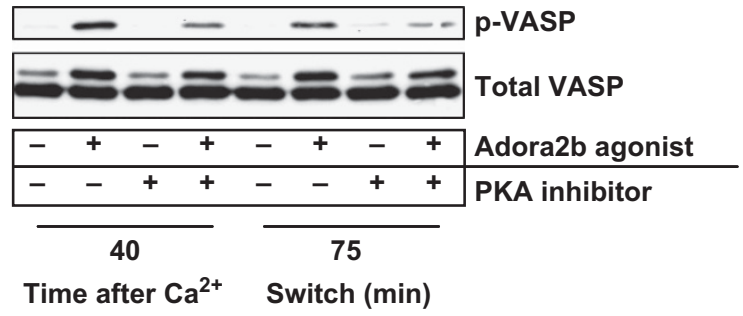

Figure 7 Epithelial vasodilator-stimulated phosphoprotein (VASP) $\left(\mathrm{Ser}^{157}\right)$ phosphorylation is induced by Adora2b (A2B adenosine receptor) signaling through protein kinase A (PKA). (a) T84 intestinal epithelial cells were pretreated on both the basolateral and apical aspect of the cell monolayer with the PKA inhibitor $(\mathrm{H} 89,30 \mu \mathrm{m})$ for $45 \mathrm{~min}$ before $5 \mathrm{~min}$ treatment with increasing concentrations of the Adora2b-specific agonist (BAY 60-6583; 1 and $10 \mu \mathrm{m}$ ) in the presence or absence of the PKA inhibitor. Cells were harvested, total protein extracted, and p-VASP $\left(\mathrm{Ser}^{157}\right)$ and total VASP levels were determined by Western blot analysis. (b) Calcium switch assays were performed using T84 intestinal epithelial cells with a fully competent barrier. Cells were placed in calcium-depleting conditions until barrier function was fully compromised and subsequently allowed to recover in calcium complete media for the time points outlined. Cells were treated on both the basolateral and apical aspect with the Adora2b-specific agonist (BAY 60-6583; $10 \mu \mathrm{m}$ ) in the presence or absence of the PKA inhibitor $(\mathrm{H} 89,30 \mu \mathrm{m})$ from the beginning of the recovery period for the time points indicated. Cells were harvested, total protein extracted, and p-VASP $\left(\mathrm{Ser}^{157}\right.$ ) and total VASP levels were determined by Western blot analysis. Results are representative of three independent experiments with 2-3 wells per time point per experiment.

source of p-VASP $\left(\mathrm{Ser}^{157}\right)$ staining in the colon was in E-cadherin-positive cells, indicating that p-VASP $\left(\mathrm{Ser}^{157}\right)$ is enriched in the colonic epithelium. In particular, p-VASP $\left(S^{157}\right)$ expression was largely observed at the E-cadherinpositive epithelial cell junctions during DSS (Figure 8a, b). A profound loss of p-VASP $\left(\mathrm{Ser}^{157}\right)$ in E-cadherin-positive epithelial cell junctions was noted in Adora2 $b^{f l / f l}$ VillinCre $^{+}$ compared with VillinCre ${ }^{+}$mice at both 4 and 7 days after DSS (Figure 8a, b, respectively). Differences were quantified by relating the percentage of p-VASP $\left(\mathrm{Ser}^{157}\right)$ staining in E-cadherin-positive areas of the tissue (Figure 8c). Our data reveal a profound reduction in VASP $\left(\mathrm{Ser}^{157}\right.$ ) phosphorylation in epithelial cells in Adora2b $b^{f l f l}$ VillinCre $^{+}$ mice compared with VillinCre ${ }^{+}$mice in DSS.

\section{An Adora2b agonist induces epithelial VASP $\left(\mathrm{Ser}^{157}\right)$ phosphorylation during DSS colitis}

We demonstrated that continuous administration of an Adora2b-specific agonist protects the intestinal epithelial barrier during acute DSS colitis (Figure 4). In vitro analysis indicates that Adora2b agonist-mediated induction of VASP $\left(\mathrm{Ser}^{157}\right)$ phosphorylation assists in rapid repair of the intestinal 
a
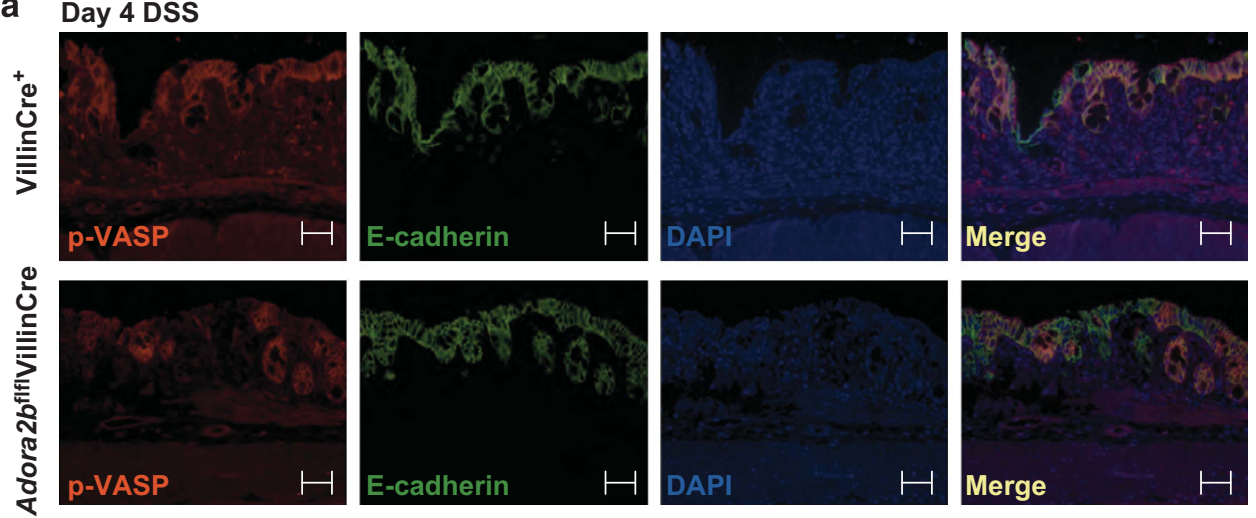

\section{b Day 7 DSS}
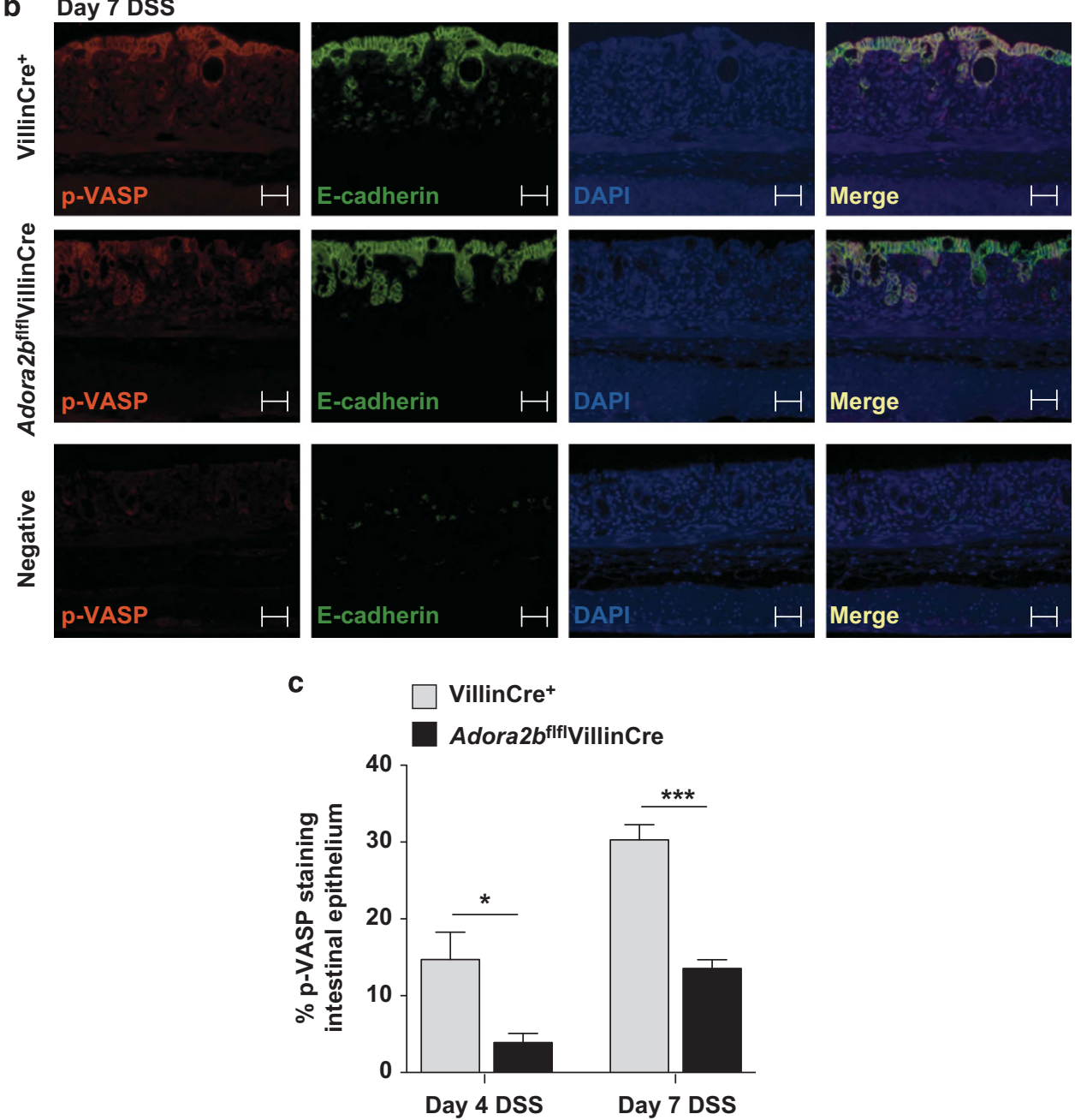

Figure 8 Loss of epithelial Adora2b (A2B adenosine receptor) expression results in significantly reduced localization of p-VASP (phosphorylated vasodilator-stimulated phosphoprotein) $\left(\mathrm{Ser}^{157}\right)$ to the intestinal epithelial barrier during acute colitis. Mice with intestinal epithelial-specific deletion of

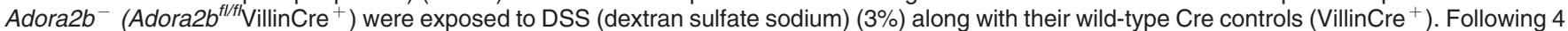
and 7 days DSS, colons were harvested by blunt dissection and formalin fixed. (a and $\mathbf{b})$ Formalin-fixed colons were deparaffinized and rehydrated. Slides were costained with p-VASP $\left(\mathrm{Ser}^{157}\right)$ and E-cadherin antibodies and appropriate fluorescent secondary antibodies. DAPI (4',6-diamidino-2phenylindole) served as a nuclear counterstain. Omission of primary antibodies was used as a negative control. Representative images were acquired at original magnification $\times 20$. Bar $=50 \mu \mathrm{m}$. (c) p-VASP $\left(\mathrm{Ser}^{157}\right)$ costaining of E-cadherin-positive cells was calculated using Olympus software $($ Shinjuku, Tokyo, Japan). Image analysis was performed in a blinded manner using images acquired at the same settings, with a defined threshold of intensity applied to all images. Results represent the percentage staining of three identically sized randomly selected E-cadherin-positive regions of interest from each image. Day 4 results represent one image per mouse with four mice in each group. Day 7 results represent 4-5 images per mouse with 5-7 mice per group. Data are displayed as the mean \pm s.e.m. Statistical analysis was performed using the Student's $t$-test. ${ }^{*} P<0.05$ and ${ }^{* * *} P<0.0001$. 

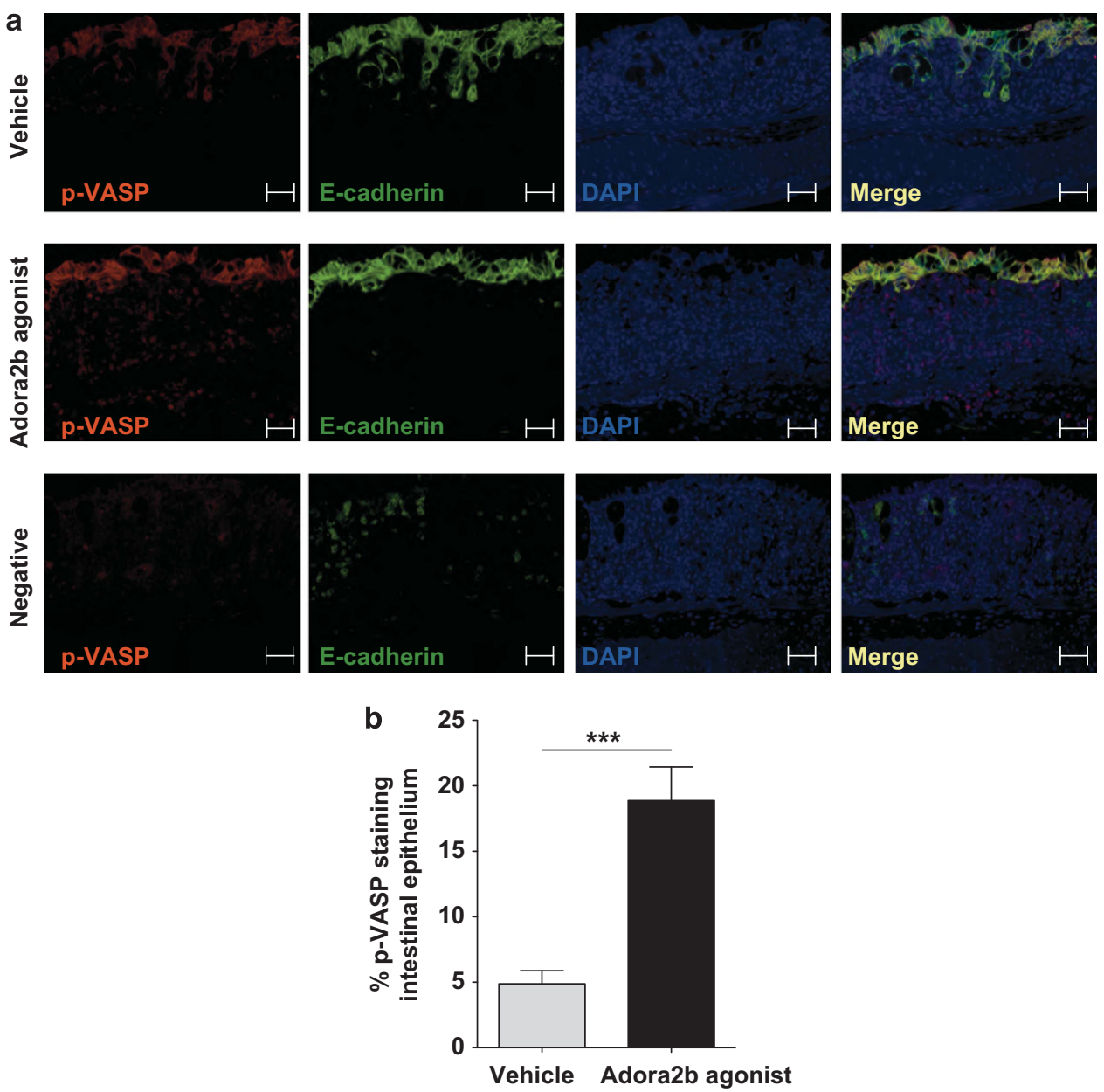

Figure 9 Adora2b (A2B adenosine receptor) agonist treatment results in significantly increased localization of $p$-VASP (phosphorylated vasodilatorstimulated phosphoprotein) (Ser ${ }^{157}$ ) to the intestinal epithelial barrier in vivo during DSS (dextran sulfate sodium) colitis. Gender-, age-, and weightmatched C57BL/6 mice were treated with an Adora2b-specific agonist (BAY 60-6583; $1.2-1.25 \mathrm{mg} / \mathrm{kg}^{-1}$ per day) or vehicle for 6 days during DSS as described in Figure 4. Colons were harvested by blunt dissection and formalin fixed. (a) Formalin-fixed colons were deparaffinized and rehydrated. Sections were costained with p-VASP $\left(\right.$ Ser $^{157}$ ) and E-cadherin antibodies as described in Figure $\mathbf{7 a}$ and $\mathbf{b}$. Representative images were acquired at original magnification $\times 20$. Bar $=50 \mu \mathrm{m}$. (b) $p$-VASP $\left(\mathrm{Ser}^{157}\right)$ staining of $\mathrm{E}$-cadherin-positive cells was calculated as described in Figure $7 \mathrm{c}$. Results are representative of 1-3 images per mouse with 5-7 mice per group. Statistical analysis was performed using the Student's $t$-test. Data are displayed as the mean \pm s.e.m. ${ }^{* * *} P<0.0001$.

epithelial barrier (Figure 6c, d). We therefore analyzed p-VASP $\left(\mathrm{Ser}^{157}\right)$ expression in vivo following Adora2b agonist treatment during DSS (Figure 9). Immunofluorescence analysis was performed in colonic tissue following vehicle or Adora2b agonist administration for 6 days during DSS, as discussed above (Figure 4). Adora2b agonist-treated mice exhibited a significantly higher level of p-VASP $\left(\mathrm{Ser}^{157}\right)$ expression in E-cadherin-positive cells in comparison with vehicle-treated mice (Figure 9a). A large portion of epithelial p-VASP $\left(\operatorname{Ser}^{157}\right)$ was observed to localize to E-cadherin-positive epithelial cell junctions following Adora2b agonist treatment (Figure 9a). p-VASP $\left(\mathrm{Ser}^{157}\right)$ levels in the epithelium were quantified by measuring the percentage of staining in standardized sections of E-cadherin-positive tissue (Figure 9b). These findings indicate that an Adora2b agonist can induce epithelial VASP $\left(\operatorname{Ser}^{157}\right)$ phosphorylation to facilitate a barrier-protective response in DSS colitis.

\section{DISCUSSION}

Current studies point to the key role played by dysregulated mucosal homeostasis in the development of IBD. ${ }^{1}$ As such, better understanding of mucosal responses in the context of IBD is of great relevance to the identification of novel therapeutic options.

The release of adenosine from inflamed tissues and its receptor signaling pathways have been highlighted as an endogenous mechanism to dampen inflammation. ${ }^{3}$ In the context of IBD, maintaining high tissue levels of adenosine has been demonstrated to protect the intestinal mucosa. ${ }^{14-16}$ However, the predominant adenosine receptor signaling pathway that mediates protection during IBD remains to be elucidated. There is evidence that adenosine signaling through the Adora2a receptor is protective in a T-cell-mediated murine model of IBD. ${ }^{17,38}$ However, an Adora2a agonist was ineffective in an acute colitis model, indicating a potential tissue- and 
context-specific role for adenosine signaling in the inflamed intestine. ${ }^{39}$ This is further highlighted when considering the potential role of the Adora2b adenosine receptor in IBD. To date, studies have indicated both a protective ${ }^{12,18}$ and deleterious effect ${ }^{19-21}$ of Adora2b receptor signaling at the level of the intestinal epithelium in murine models of colitis. Divergent results observed may be because of specific effects of Adora2b signaling at alternative stromal tissue sites. Indeed vascular endothelial expression of the Adora2b receptor has been demonstrated to be an important protective pathway in another model of acute mucosal inflammation. ${ }^{8}$ Therefore, we were interested in defining the Adora $2 \mathrm{~b}$ signaling events occurring on stromal tissue during acute intestinal inflammation. We postulated that the Adora $2 \mathrm{~b}$ receptor had a tissuespecific role to play during the development of IBD.

To dissect the role of Adora $2 \mathrm{~b}$ on stromal tissue during acute colitis, we examined mice lacking the Adora2b receptor $\left(\right.$ Adora $2 b^{-l-}$ ) over a time course of DSS (Figure 1). We observed significant increases in disease activity parameters early in the time course of disease in Adora $2 b^{-1-}$ mice compared with wild-type controls (Figure 1). As early as day 3 , Adora $2 b^{-1-}$ mice exhibited pronounced colonic shortening and loss of epithelial barrier function (Figure 1b, c, respectively). This culminated in considerably greater histological disease (Figure 1e-f) and robust recruitment of neutrophils to the colonic lamina propria of $A d o r a 2 b^{-1-}$ mice in comparison with wild-type controls (Figure 1d). Taken together, our findings suggest a protective role for Adora2b signaling during acute intestinal inflammation.

Previous findings in a model of hypoxia-driven inflammation indicate that Adora2b receptors on the vascular endothelium mediate a protective response in acute inflammation. ${ }^{8}$ Using a novel Adora2b-flox mouse line, we generated mice with tissue-specific deletion of Adora2b on the vascular endothelium. These mice did not exhibit any alteration in disease outcome compared with their genetic controls during DSS colitis (Figure 2), prompting us to consider an alternative stromal source of Adora $2 \mathrm{~b}$-mediated mucosal protection.

Intestinal epithelial cells express a high level of the Adora2b receptor, which can be upregulated by mediators associated with colitis. ${ }^{22}$ Using a tissue-specific gene approach, we demonstrated that genetic loss of Adora2b in intestinal epithelial cells led to significantly greater disease activity during DSS (Figure 3). As in whole-body Adora2b deletion (Figure 1), we observed pronounced early onset of disease in mice lacking epithelial Adora2b (Adora2b $b^{f l f l}$ VillinCre) culminating in worsened DSS colitis outcome in Adora2b $b^{f l / f l}$ VillinCre mice in all parameters measured (Figure 3).

Given our current findings, we hypothesized that pharmacologic targeting of the Adora2b receptor may be of therapeutic benefit in acute colitis. Mice administered an Adora2b-specific agonist were profoundly protected from development of DSS colitis (Figure 4). Importantly, Adora2b agonist treatment improved intestinal epithelial barrier function (Figure 4c), suggesting agonist-directed mucosal protection in acute colitis. While previous studies illustrated that an Adora $2 \mathrm{~b}$ antagonist is deleterious in colitis, ${ }^{12}$ this is the first study demonstrating that Adora $2 \mathrm{~b}$ signaling can be therapeutically targeted for tissue protection in a model of IBD.

Our observations to date suggest that endogenous epithelial Adora $2 \mathrm{~b}$ is a barrier-protective signaling pathway in acute colitis. We therefore concentrated our efforts on elucidating the functional role of the Adora2b receptor on the intestinal epithelium during the acute response in DSS. Generally, DSS colitis is characterized by elevated tissue cytokine levels, enhanced epithelial cell apoptosis, and loss of intestinal barrier function. ${ }^{28,40,41}$ We pursued studies to interrogate the role of the Adora2b receptor in processes known to be involved in these features of early mucosal barrier dysfunction. Previous study suggests that Adora2b signaling induces the release of the proinflammatory cytokine, IL- $6,{ }^{42}$ and the anti-inflammatory cytokine, IL-10, ${ }^{12}$ from the intestinal epithelium. Adora $2 b^{f l f l}$ VillinCre mice were observed to have a slightly elevated tissue content of IL- 6 but no significant alteration in IL-10 compared with control mice (Figure 3d). In contrast, Adora2b agonisttreated mice exhibited a significant decrease in IL-6 tissue levels and a reduction in IL-10 compared with vehicle controls (Figure 4d). Taking these findings in the context of the previous studies mentioned, our results suggest that direct regulation of cytokine release at the epithelium was not the predominant function of the epithelial Adora2b receptor in our model. We next investigated the potential role of Adora2b receptor signaling in mechanisms that promote epithelial barrier breakdown during DSS colitis. However, we failed to observe an effect of Adora2b signaling on cytokine-induced epithelial barrier permeability, MLC phosphorylation, $\mathrm{NF}-\kappa \mathrm{B}$ transcriptional activation, epithelial cell apoptosis (Figure 5a-d, respectively), or mucin-2 expression (Supplementary Figure S3). These studies were predominantly performed in vitro and were used to direct additional in vitro and in vivo studies. We cannot abandon the possibility that one of the mechanisms excluded by our in vitro studies may contribute to the phenotype we observe in our animal model.

Having ruled out direct effects of epithelial Adora $2 b$ receptor signaling on epithelial cytokine release and processes implicated in epithelial barrier breakdown, we considered a potential role for the Adora2b in mechanisms involved in epithelial barrier repair. To do this, we used an in vitro model with polarized intestinal epithelial cells. First, we confirmed the capability of the Adora2b-specific agonist to induce known receptor second messenger pathways and demonstrated efficient increase in intracellular cAMP following agonist treatment (Supplementary Figure S4). We subsequently generated genetic knockout of Adora $b$ in intestinal epithelial cells to mirror our in vivo model (Supplementary Figure S5b). To model epithelial tight junction rearrangement and barrier dysfunction observed in IBD, we used a calcium switch assay in vitro. ${ }^{36,37}$ Importantly, in this model epithelial junction reassembly and repair can be studied. Adora2b KD cells exhibited impaired recovery of barrier function following junction disassembly (Figure 6a), whereas agonist treatment during recovery significantly enhanced the rate of barrier 
restitution when compared with vehicle treatment (Figure 6c). Considering our observations that Adora2b signaling was capable of enhancing barrier recovery (Figure 6) and also elevated intracellular cAMP (Supplementary Figure S4), we noted that cAMP-mediated phosphorylation of VASP had previously been implicated in recovery of epithelial barrier function. ${ }^{37}$ In this study, expression of VASP in the intestinal epithelium was demonstrated to be necessary for the recovery of epithelial barrier function, with phosphorylated VASP (p-VASP Ser ${ }^{157}$ ) localizing to the junction during barrier recovery. Using these previous findings, we examined our Adora2b-deficient cells and demonstrated an appreciable decrease in the level of p-VASP $\left(\mathrm{Ser}^{157}\right)$ during barrier recovery compared with control cells (Figure 6b). In addition, Adora2b agonist treatment of wild-type cells enhanced p-VASP $\left(\mathrm{Ser}^{157}\right)$ expression during barrier recovery over that in vehicle-treated cells (Figure 6d). Importantly, an increased level of p-VASP $\left(\mathrm{Ser}^{157}\right)$ was observed to colocalize with E-cadherin within 30 min of recovery following agonist treatment compared with vehicle, suggesting an accumulation of this protein at the repairing junction (Figure 6e). Using a PKA antagonist, we demonstrated that induction of PKA was a major pathway by which the Adora2b receptor induced VASP $\left(\mathrm{Ser}^{157}\right.$ ) phosphorylation either in intact or repairing epithelial cells (Figure 7).

Having identified the phosphorylation of VASP $\left(\mathrm{Ser}^{157}\right)$ as a likely mechanism by which the Adora2b receptor repairs the intestinal epithelial barrier in vitro, we wanted to investigate the potential relevance of this in vivo. Adora $2 b^{f l f l}$ VillinCre mice demonstrated a significantly diminished expression of p-VASP $\left(\operatorname{Ser}^{157}\right)$, particularly at epithelial cell junctions during the early onset of DSS (day 4; Figure 8a). This was also the case following the complete time course of DSS (day 7; Figure 8b). Early loss of p-VASP $\left(\mathrm{Ser}^{157}\right)$ in the epithelial junctions coincided with our observation of enhanced epithelial barrier permeability in Adora $2 b^{f l f l}$ VillinCre mice compared with control mice (Figure 3c). In contrast, mice treated with the Adora2b agonist during DSS were observed to have a pronounced increase in p-VASP $\left(\mathrm{Ser}^{157}\right)$ at epithelial cell junctions compared with vehicle-treated controls (Figure 9). This matched with our finding of significantly reduced epithelial barrier permeability in agonist-treated mice compared with vehicle controls at the same time point (Figure 4c). These observations suggest that Adora2b signaling in the intestinal epithelium in vivo regulates VASP $\left(\mathrm{Ser}^{157}\right)$ phosphorylation to enhance epithelial barrier function during acute inflammation. Of note, we did not analyze the expression of other junctional proteins in these mice and therefore cannot exclude the possibility that in vivo Adora2b signaling may have additional effects on epithelial junction proteins.

In this study, we used a whole-body and tissue-specific genetic approach to define the contribution of Adora2b to mucosal inflammation. Initial studies in mice with whole-body deletion of Adora2b (Adora2b $b^{-1-}$ ) demonstrated rapid, early onset of mucosal inflammation in Adora $2 b^{-1-}$ mice during experimental colitis. Excitingly, we determined that intestinal epithelial-specific Adora2b signaling is a key pathway in mediating mucosal barrier protection during acute colitis. In vitro and in vivo analysis points to Adora $2 \mathrm{~b}$ phosphorylation of VASP $\left(\mathrm{Ser}^{157}\right)$ and its localization to epithelial cell junctions as a potential mechanism by which endogenous Adora2b mediates a barrier protective effect. Herein, we present novel studies demonstrating for the first time that Adora $2 \mathrm{~b}$ signaling can be exploited as a therapeutic pathway in a model of IBD. Taken together, we have defined that specific signaling through intestinal-epithelial Adora2b receptors provides potent protection during acute intestinal inflammation, an important finding that may be exploited as a novel therapeutic strategy in IBD.

\section{METHODS}

DSS colitis. Adora2b-deficient mice, ${ }^{8}$ mice with tissue-specific $A d o r a 2 b$ deletion (for more details see Supplementary Materials), matched genetic controls, or C57BL/6 mice were used in DSS studies (3-4.5\%), as described previously. ${ }^{13,44,45}$ Adora2b agonist (BAY 606583; Tocris Bioscience, Bristol, UK) or vehicle (30\% SolutolHS15 in $0.9 \%$ saline; BASF, Florham Park, NJ) was administered by subcutaneous osmotic pump (Alzet; DURECT Corporation, Cupertino, CA) at $1.2-1.25 \mathrm{mg} \mathrm{kg}^{-1}$ mouse per day.

Flow cytometric analysis. Flow cytometric analysis of lamina propria leukocytes was performed with antibodies against GR-1 (RB6-8C5), SiglecF (E50-2440), F4/80 (BM8), CD11c (N418), MHCII (M5/ 114.15.2), and CD45 (30-F11), as outlined previously. ${ }^{13,43}$

In vivo permeability. Tissue permeability was measured using FITC-labeled dextran as described previously. ${ }^{13}$

Cell culture and generation of short hairpin RNA KD. Cells were cultured as outlined in Supplementary Materials. ${ }^{30,46-48}$ Constitutive $\mathrm{KD}$ of Adora $2 b$ was generated using MISSION shRNA technology (as described in Supplementary Materials).

In vitro FITC-dextran flux assay. FITC-dextran $(3 \mathrm{kDa})$ flux assays were performed in T84 intestinal epithelial cells following cytomix treatment (TNF $\alpha, \mathrm{IL}-1 \beta$, and IFN $\gamma ; 10 \mathrm{ng} / \mathrm{ml})$ in the presence of vehicle or Adora2b agonist (BAY 60-6583, $10 \mu \mathrm{M}$ ) as described ${ }^{13,49}$ (for more details see Supplementary Materials).

Luciferase reporter assay. Caco-2 intestinal epithelial cells were transfected with equal amounts of NF- $\kappa \mathrm{B}$ luciferase and Renilla luciferase plasmids. Following $24 \mathrm{~h}$, cells were treated with vehicle, $\mathrm{TNF} \alpha(10 \mathrm{ng} / \mathrm{ml})$, the Adora2b agonist alone (BAY 60-6583, $10 \mu \mathrm{M}$ ), or in combination with TNF $\alpha$. Luminescence was detected as described in Supplementary Materials.

TUNEL staining. Apoptotic epithelial cells were detected by TUNEL staining of formalin-fixed, paraffin-embedded tissue (for details see Supplementary Materials).

Calcium switch assay. Calcium switch assays in T84 intestinal epithelial cells were performed as outlined previously. ${ }^{36}$ Barrier restitution rate was determined by the addition of FITC-dextran $(10 \mathrm{kDa})$ to the apical aspect at the time of recovery and sampling of FITC in the basolateral compartment (for further details see Supplementary Methods).

Western blot analysis. Equal amounts of whole-cell lysates were separated by gel electrophoresis, transferred, and membranes incubated with either anti-human p-VASP $\left(\mathrm{Ser}^{157}\right.$ ) (Cell Signaling, Danvers, MA), anti-human anti-VASP (BD Transduction Laboratories, San Jose, CA), anti-human p-MLC-2 ( $\left.\operatorname{Ser}^{19}\right)$ (Cell Signaling), anti-human MLC-2 (Cell signaling), or anti-actin (Calbiochem). 
Immunofluorescence. Paraffin-embedded sections or paraformaldehyde fixed cell membranes were incubated with anti-human p-VASP $\left(\mathrm{Ser}^{157}\right)$ or anti-human E-cadherin followed by fluorescent detection.

Statistical analysis. GraphPad Prism Analysis software (GraphPad Software, La Jolla, CA) was used to perform statistical analysis. Analysis of variance (ANOVA) followed by post-test or Student's $t$-test was used where appropriate and results are expressed as mean \pm s.e.m.

For further details see Supplementary Materials.

SUPPLEMENTARY MATERIAL is linked to the online version of the paper at http://www.nature.com/mi

\section{ACKNOWLEDGMENTS}

We acknowledge technical assistance from Maria I. Wong, Kristann Magee, Melissa Ledezma, Chelsea Ruller, Caleb Kelly, Aneta Gandjeva, and Dan Koyanagi. Consultation from Simon J. Kelley was greatly appreciated. Human T84 intestinal epithelial cells were a kind gift from Stephen J. Keely, $\mathrm{PhD}$, Royal College of Surgeons Dublin. CHO wild-type cells were a gift from Twila Jackson, PhD, University of Colorado Denver. CHO-Adora2boverexpresssing cells were a gift from Prof. Bertil Fredholm, Karolinska Institute. The NF- $\kappa B$-luciferase reporter construct was kindly provided by Prof. Sean Colgan, University of Colorado Denver. The present study was supported by National Institute of Health grants R01 DK097075, R01HL092188, R01- HL098294, POI-HL114457, and R01-HL119837 and a grant from the Crohn's and Colitis Foundation (CCFA) to HKE. This work was also supported by a National Institute of Health grant K01 DK099485, the President's Research Scholar Award from the American Gastroenterological Association (AGA), and grants from the Crohn's and Colitis Foundation of America (CCFA no.s 2865 and 276536) to CMA.

\section{DISCLOSURE}

The authors declare no conflict of interest.

c 2015 Society for Mucosal Immunology

\section{REFERENCES}

1. Khor, B., Gardet, A. \& Xavier, R.J Genetics and pathogenesis of inflammatory bowel disease. Nature 474, 307-317 (2011).

2. Molodecky, N.A. et al. Increasing incidence and prevalence of the inflammatory bowel diseases with time, based on systematic review. Gastroenterology 142, 46-54 e42 (2012).

3. Aherne, C.M., Kewley, E.M. \& Eltzschig, H.K The resurgence of A2B adenosine receptor signaling. Biochim. Biophys. Acta 1808, 1329-1339 (2011).

4. Lukashev, D., Ohta, A., Apasov, S., Chen, J.F. \& Sitkovsky, M Cutting edge: physiologic attenuation of proinflammatory transcription by the Gs proteincoupled A2A adenosine receptor in vivo. J. Immunol. 173, 21-24 (2004).

5. Impellizzeri, D. et al. CGS 21680, an agonist of the adenosine (A2A) receptor, decreases acute lung inflammation. Eur. J. Pharmacol. 668, 305-316 (2011).

6. Chouker, A. et al. Critical role of hypoxia and A2A adenosine receptors in liver tissue-protecting physiological anti-inflammatory pathway. Mol. Med. 14, 116-123 (2008).

7. Ohta, A. \& Sitkovsky, M Role of G-protein-coupled adenosine receptors in downregulation of inflammation and protection from tissue damage. Nature 414, 916-920 (2001).

8. Eckle, T. etal. A2B adenosine receptor dampens hypoxia-induced vascular leak. Blood 111, 2024-2035 (2008).

9. Csoka, B. et al. A2B adenosine receptors protect against sepsis-induced mortality by dampening excessive inflammation. J. Immunol. 185, 542-550 (2010).

10. Koeppen, M. et al. Adora2b signaling on bone marrow derived cells dampens myocardial ischemia-reperfusion injury. Anesthesiology 116, 1245-1257 (2012).

11. Schingnitz, U. et al. Signaling through the A2B adenosine receptor dampens endotoxin-induced acute lung injury. J. Immunol. 184, 5271-5279 (2010).
12. Frick, J.S. et al. Contribution of adenosine A2B receptors to inflammatory parameters of experimental colitis. J. Immunol. 182, 4957-4964 (2009).

13. Aherne, C.M. et al. Neuronal guidance molecule netrin-1 attenuates inflammatory cell trafficking during acute experimental colitis. Gut 61, 695-705 (2012).

14. Louis, N.A. et al. Control of IFN-alphaA by CD73: implications for mucosal inflammation. J. Immunol. 180, 4246-4255 (2008).

15. Friedman, D.J. et al. From the Cover: CD39 deletion exacerbates experimental murine colitis and human polymorphisms increase susceptibility to inflammatory bowel disease. Proc. Natl. Acad. Sci. USA 106, 16788-16793 (2009).

16. Antonioli, L. et al. Inhibition of adenosine deaminase attenuates inflammation in experimental colitis. J. Pharmacol. Exp. Ther. 322, 435-442 (2007).

17. Naganuma, M. et al. Cutting edge: critical role for A2A adenosine receptors in the T cell-mediated regulation of colitis. J. Immunol. 177, 2765-2769 (2006).

18. Alam, M., Wilson, J. \& Ernst, P Role of adenosine A2B receptors in regulating inflammatory responses of TNBS-induced experimental colitis. J. Immunol. 184, 87.2 (2010).

19. Kolachala, V.L. et al. A2B adenosine receptor gene deletion attenuates murine colitis. Gastroenterology 135, 861-870 (2008).

20. Kolachala, V. et al. Blockade of adenosine A2B receptors ameliorates murine colitis. Br. J. Pharmacol. 155, 127-137 (2008).

21. Ingersoll, S.A. et al. A((2)B)AR expression in non-immune cells plays an important role in the development of murine colitis. Dig. Liver Dis. 44, 819-826 (2012).

22. Kolachala, V. et al. TNF-alpha upregulates adenosine 2b (A2b) receptor expression and signaling in intestinal epithelial cells: a basis for A2bR overexpression in colitis. Cell. Mol. Life Sci. 62, 2647-2657 (2005).

23. Eckle, T. et al. Cardioprotection by ecto-5'-nucleotidase (CD73) and A2B adenosine receptors. Circulation 115, 1581-1590 (2007).

24. Baraldi, P.G., Tabrizi, M.A., Fruttarolo, F., Romagnoli, R. \& Preti, D Recent improvements in the development of $\mathrm{A}(2 \mathrm{~B})$ adenosine receptor agonists. Purinergic Signal. 4, 287-303 (2008).

25. Ivanov, A.I., Parkos, C.A. \& Nusrat, A Cytoskeletal regulation of epithelial barrier function during inflammation. Am. J. Pathol. 177, 512-524 (2010).

26. Su, L. et al. TNFR2 activates MLCK-dependent tight junction dysregulation to cause apoptosis-mediated barrier loss and experimental colitis. Gastroenterology 145, 407-415 (2013).

27. Xavier, R.J. \& Podolsky, D.K Unravelling the pathogenesis of inflammatory bowel disease. Nature 448, 427-434 (2007).

28. Araki, Y., Mukaisyo, K., Sugihara, H., Fujiyama, Y. \& Hattori, T Increased apoptosis and decreased proliferation of colonic epithelium in dextran sulfate sodium-induced colitis in mice. Oncol. Rep. 24, 869-874 (2010).

29. Van der Sluis, M. et al. Muc2-deficient mice spontaneously develop colitis, indicating that MUC2 is critical for colonic protection. Gastroenterology 131, 117-129 (2006).

30. Khoury, J., Ibla, J.C., Neish, A.S. \& Colgan, S.P Antiinflammatory adaptation to hypoxia through adenosine-mediated cullin-1 deneddylation. J. Clin. Invest. 117, 703-711 (2007).

31. Turner, J.R. et al. Physiological regulation of epithelial tight junctions is associated with myosin light-chain phosphorylation. Am. J. Physiol. 273, C1378-C1385 (1997).

32. Majumdar, S. \& Aggarwal, B.B Adenosine suppresses activation of nuclear factor-kappaB selectively induced by tumor necrosis factor in different cell types. Oncogene 22, 1206-1218 (2003).

33. Bergmann, S. et al. Adenosine and homocysteine together enhance TNFmediated cytotoxicity but do not alter activation of nuclear factor-kappa B in L929 cells. J. Immunol. 153, 1736-1743 (1994).

34. Tambuwala, M.M. et al. Loss of prolyl hydroxylase-1 protects against colitis through reduced epithelial cell apoptosis and increased barrier function. Gastroenterology 139, 2093-2101 (2010).

35. McNamara, N. et al. Adenosine up-regulation of the mucin gene, MUC2, in asthma. FASEB J. 18, 1770-1772 (2004).

36. Parkos, C.A. et al. Intestinal epithelia (T84) possess basolateral ligands for CD11b/CD18-mediated neutrophil adherence. Am. J. Physiol. 268, C472-C479 (1995).

37. Lawrence, D.W., Comerford, K.M. \& Colgan, S.P Role of VASP in reestablishment of epithelial tight junction assembly after $\mathrm{Ca}^{2+}$ switch. Am. J. Physiol. Cell Physiol. 282, C1235-C1245 (2002). 


\section{ARTICLES}

38. Odashima, M. et al. Activation of A2A adenosine receptor attenuates intestinal inflammation in animal models of inflammatory bowel disease. Gastroenterology 129, 26-33 (2005).

39. Selmeczy, Z., Csoka, B., Pacher, P., Vizi, E.S. \& Hasko, G The adenosine A2A receptor agonist CGS 21680 fails to ameliorate the course of dextran sulphate-induced colitis in mice. Inflamm. Res. 56, 204-209 (2007).

40. Yan, Y. et al. Temporal and spatial analysis of clinical and molecular parameters in dextran sodium sulfate induced colitis. PLoS One 4, e6073 (2009).

41. Alex, P. et al. Distinct cytokine patterns identified from multiplex profiles of murine DSS and TNBS-induced colitis. Inflamm. Bowel Dis. 15, 341-352 (2009).

42. Sitaraman, S.V. et al. Neutrophil-epithelial crosstalk at the intestinal lumenal surface mediated by reciprocal secretion of adenosine and IL-6. J. Clin. Invest. 107, 861-869 (2001).

43. Collins, C.B. et al. Inhibition of N-terminal ATPase on HSP9O attenuates colitis through enhanced Treg function. Mucosal Immunol. 6, 960-971 (2013).
44. Siegmund, B. et al. Neutralization of interleukin-18 reduces severity in murine colitis and intestinal IFN-gamma and TNF-alpha production. Am. J. Physiol. Regul. Integr. Comp. Physiol. 281, R1264-R1273 (2001).

45. Burns, R.C. et al. Antibody blockade of ICAM-1 and VCAM-1 ameliorates inflammation in the SAMP-1/Yit adoptive transfer model of Crohn's disease in mice. Gastroenterology 121, 1428-1436 (2001).

46. Schulte, G. \& Fredholm, B.B Human adenosine $A(1), A(2 A), A(2 B)$, and $A(3)$ receptors expressed in Chinese hamster ovary cells all mediate the phosphorylation of extracellular-regulated kinase 1/2. Mol. Pharmacol. 58, 477-482 (2000).

47. Haeberle, H.A. et al. Oxygen-independent stabilization of hypoxia inducible factor (HIF)-1 during RSV infection. PLoS One 3, e3352 (2008).

48. Gessi, S. et al. Adenosine receptors in colon carcinoma tissues and colon tumoral cell lines: focus on the A(3) adenosine subtype. J. Cell Physiol. 211, 826-836 (2007).

49. Keely, S. et al. Dexamethasone-pDMAEMA polymeric conjugates reduce inflammatory biomarkers in human intestinal epithelial monolayers. J. Control Rel. 135, 35-43 (2009). 